TRANSACTIONS OF THE

AMERICAN MATHEMATICAL SOCIETY

Volume 352, Number 3 , Pages 1071-1111

S $0002-9947(99) 02275-8$

Article electronically published on September 9, 1999

\title{
ON THE DISTRIBUTION OF POINTS IN PROJECTIVE SPACE OF BOUNDED HEIGHT
}

\author{
KWOK-KWONG CHOI
}

\begin{abstract}
In this paper we consider the uniform distribution of points in compact metric spaces. We assume that there exists a probability measure on the Borel subsets of the space which is invariant under a suitable group of isometries. In this setting we prove the analogue of Weyl's criterion and the Erdös-Turán inequality by using orthogonal polynomials associated with the space and the measure. In particular, we discuss the special case of projective space over completions of number fields in some detail. An invariant measure in these projective spaces is introduced, and the explicit formulas for the orthogonal polynomials in this case are given. Finally, using the analogous Erdös-Turán inequality, we prove that the set of all projective points over the number field with bounded Arakelov height is uniformly distributed with respect to the invariant measure as the bound increases.
\end{abstract}

\section{INTRODUCTION}

Let $k$ be an algebraic number field, $v$ a place of $k$ and $k_{v}$ the completion of $k$ with respect to $v$. Let $\|\cdot\|_{v}$ be an absolute value from $v$ which extends the Euclidean absolute value on $k_{v}$ if $v \mid \infty$ and the usual $p$-adic absolute value if $v \mid p$. We also use a second absolute value, determined by

$$
|\cdot|_{v}:=\|\cdot\|_{v}^{\frac{d_{v}}{d}}
$$

where $d=[k: \mathbb{Q}]$ and $d_{v}=\left[k_{v}: \mathbb{Q}_{v}\right]$. We note that the product formula holds for the absolute values $|\cdot|_{v}$. We extend both absolute values to a norm on finite dimensional vector spaces over $k_{v}$ as follows. For any column vector

$$
\boldsymbol{\alpha}=\left(\begin{array}{c}
\alpha_{0} \\
\alpha_{1} \\
\vdots \\
\alpha_{N-1}
\end{array}\right)
$$

in $k_{v}^{N}$, define

$$
\|\boldsymbol{\alpha}\|_{v}:= \begin{cases}\left\{\sum_{j=0}^{N-1}\left\|\alpha_{j}\right\|_{v}^{2}\right\}^{1 / 2} & \text { if } v \mid \infty \\ \max _{0 \leq j \leq N-1}\left\|\alpha_{j}\right\|_{v} & \text { if } v \nmid \infty\end{cases}
$$

and

$$
|\boldsymbol{\alpha}|_{v}:=\|\boldsymbol{\alpha}\|_{v}^{\frac{d_{v}}{d}},
$$

in both the infinite and finite cases.

Received by the editors April 24, 1997 and, in revised form, December 18, 1997.

1991 Mathematics Subject Classification. Primary 11J61, 11J71, 11K60.

The author was supported by NSF Grant DMS 9304580. 
Let $\mathbb{P}^{N-1}\left(k_{v}\right)$ denote the $N$-dimensional projective space over $k_{v}$ and write $\left[\alpha_{0}, \alpha_{1}, \cdots, \alpha_{N-1}\right]$ for the homogeneous coordinates of a generic element in $\mathbb{P}^{N-1}\left(k_{v}\right)$. We let the quotient map $\phi: k_{v}^{N}-\{\mathbf{0}\} \longrightarrow \mathbb{P}^{N-1}\left(k_{v}\right)$ be

$$
\phi\left(\alpha_{0}, \alpha_{1}, \cdots, \alpha_{N-1}\right):=\left[\alpha_{0}, \alpha_{1}, \cdots, \alpha_{N-1}\right] .
$$

As in [1], [5] and [9], one can define a projective metric on $\mathbb{P}^{N-1}\left(k_{v}\right)$ as follows. If $\boldsymbol{\alpha}$ and $\boldsymbol{\beta}$ belong to $\mathbb{P}^{N-1}\left(k_{v}\right)$, then we define

$$
\Delta_{v}(\boldsymbol{\alpha}, \boldsymbol{\beta}):=\frac{\|\boldsymbol{\alpha} \wedge \boldsymbol{\beta}\|_{v}}{\|\boldsymbol{\alpha}\|_{v}\|\boldsymbol{\beta}\|_{v}}
$$

and

$$
\delta_{v}(\boldsymbol{\alpha}, \boldsymbol{\beta}):=\frac{|\boldsymbol{\alpha} \wedge \boldsymbol{\beta}|_{v}}{|\boldsymbol{\alpha}|_{v}|\boldsymbol{\beta}|_{v}},
$$

where $\wedge$ is the wedge product. It follows from (1.2) that

$$
\delta_{v}(\boldsymbol{\alpha}, \boldsymbol{\beta})=\Delta_{v}(\boldsymbol{\alpha}, \boldsymbol{\beta})^{\frac{d_{v}}{d}} .
$$

Clearly, the projective metrics are well-defined on $\mathbb{P}^{N-1}\left(k_{v}\right)$. It can also be shown that the induced metric topology coincides with the quotient topology determined by $\phi$.

For any $M \times N$ matrix $A$ over $k_{v}$, we extend $|\cdot|_{v}$ to $A$ by setting

$$
|A|_{v}:=\sup \left\{|A \boldsymbol{\alpha}|_{v}: \boldsymbol{\alpha} \in k_{v}^{N},|\boldsymbol{\alpha}|_{v} \leq 1\right\} .
$$

If $v \nmid \infty$ and $A=\left(a_{i j}\right)$, we find that

$$
|A|_{v}=\max \left\{\left|a_{i j}\right|_{v}: 1 \leq i \leq M, 1 \leq j \leq N\right\} .
$$

If $v \mid \infty$, let $A^{*}$ denote the complex conjugate transpose of $A$ and let

$$
0 \leq \lambda_{1} \leq \lambda_{2} \leq \cdots \leq \lambda_{N}
$$

denote the eigenvalues of the positive semi-definite matrix $A^{*} A$. Then we have

$$
|A|_{v}=\lambda_{N}^{\frac{d_{v}}{2 d}} .
$$

Let $\operatorname{GL}\left(N, k_{v}\right)$ and $\operatorname{PGL}\left(N, k_{v}\right)$ be the general linear group and the projective general linear group of $N \times N$ non-singular matrices over $k_{v}$, respectively. Then we define a map $\eta_{v}: \operatorname{GL}\left(N, k_{v}\right) \longrightarrow[1, \infty)$ by

$$
\eta_{v}(A):=|A|_{v}\left|A^{-1}\right|_{v} .
$$

Since $\eta_{v}(\alpha A)=\eta_{v}(A)$ for all $\alpha \in k_{v}^{*}$, so $\eta_{v}$ is also well-defined as a map from $\operatorname{PGL}\left(N, k_{v}\right)$ into $[1, \infty)$. It was shown in [5] that for any $A$ in $\operatorname{PGL}\left(N, k_{v}\right)$ and $\boldsymbol{\alpha}, \boldsymbol{\beta}$ in $\mathbb{P}^{N-1}\left(k_{v}\right)$, we have

$$
\eta_{v}(A)^{-1} \delta_{v}(\boldsymbol{\alpha}, \boldsymbol{\beta}) \leq \delta_{v}(A \boldsymbol{\alpha}, A \boldsymbol{\beta}) \leq \eta_{v}(A) \delta_{v}(\boldsymbol{\alpha}, \boldsymbol{\beta}) .
$$

Furthermore, the identity

$$
\delta_{v}(\boldsymbol{\alpha}, \boldsymbol{\beta})=\delta_{v}(A \boldsymbol{\alpha}, A \boldsymbol{\beta})
$$

holds for all $\boldsymbol{\alpha}$ and $\boldsymbol{\beta}$ in $\mathbb{P}^{N-1}\left(k_{v}\right)$ if and only if $\eta_{v}(A)=1$. The inequalities (1.5) are best possible, and the identity (1.6) shows that the group of isometries on $\mathbb{P}^{N-1}\left(k_{v}\right)$ is given by

$$
\operatorname{ISO}\left(N, k_{v}\right):=\left\{A \in \operatorname{PGL}\left(N, k_{v}\right): \eta_{v}(A)=1\right\} .
$$


In view of $(1.4), \operatorname{ISO}\left(N, k_{v}\right)$ is also the group of isometries for $\Delta_{v}(\boldsymbol{\alpha}, \boldsymbol{\beta})$. Moreover, by writing $A^{-1}=\operatorname{adj} A / \operatorname{det} A$, it is easy to show that if $A$ is an element in $\operatorname{PGL}\left(N, k_{v}\right)$, then

$$
\frac{|A|_{v}}{|\operatorname{det} A|_{v}^{\frac{1}{N}}} \leq \eta_{v}(A) \leq \frac{|A|_{v}^{N}}{|\operatorname{det} A|_{v}}
$$

If $\boldsymbol{\beta}$ belongs to $\mathbb{P}^{N-1}(k)$, then we define its height by

$$
H(\boldsymbol{\beta})=\prod_{v}|\boldsymbol{\beta}|_{v},
$$

where the product is over all places $v$ of $k$. In view of the product formula, the height function is well-defined on $\mathbb{P}^{N-1}(k)$. To illustrate a basic Diophantine inequality in this setting, we state the following projective form of Dirichlet's Theorem (e.g. Theorem 1 of [1] or Theorem 1 of [5]).

Dirichlet's Theorem. Let $\boldsymbol{\alpha}$ belong to $\mathbb{P}^{N-1}\left(k_{v}\right), \tau$ belong to $k_{v}$, and assume that $1 \leq|\tau|_{v}$. Then there exists $\boldsymbol{\beta}$ in $\mathbb{P}^{N-1}(k)$ such that

(i) $H(\boldsymbol{\beta}) \leq c_{k}(N)|\tau|_{v}^{N-1}$,

(ii) $\delta_{v}(\boldsymbol{\alpha}, \boldsymbol{\beta}) \leq c_{k}(N)\left\{|\tau|_{v} H(\boldsymbol{\beta})\right\}^{-1}$,

where

$$
c_{k}(N):=2\left\|\Delta_{k}\right\|^{\frac{1}{2 d}} \prod_{w \mid \infty} r_{w}(N)^{\frac{d_{w}}{d}}
$$

$\Delta_{k}$ is the discriminant of $k$ and

$$
r_{w}(N):= \begin{cases}\pi^{-\frac{1}{2}}\left\{\Gamma\left(\frac{1}{2} N+1\right)\right\}^{\frac{1}{N}} & \text { if } w \text { is real, } \\ (2 \pi)^{-\frac{1}{2}}\{\Gamma(N+1)\}^{\frac{1}{2 N}} & \text { if } w \text { is complex. }\end{cases}
$$

Here $\|\cdot\|$ denotes the usual absolute value on $\mathbb{C}$. This form of Dirichlet's Theorem states that every $\boldsymbol{\alpha}$ in $\mathbb{P}^{N-1}\left(k_{v}\right)$ can be well approximated by a rational point $\boldsymbol{\beta}$ in $\mathbb{P}^{N-1}(k)$ with low height $H(\boldsymbol{\beta})$. So it suggests that with respect to a suitable measure, the rational points with low height should be distributed quite uniformly. Our main objective in this paper is to show that the set $\left\{\boldsymbol{\beta} \in \mathbb{P}^{N-1}(k): H(\boldsymbol{\beta}) \leq H\right\}$ as $H \longrightarrow \infty$ is $\mu_{v}^{N}$-uniformly distributed, where the invariant measure $\mu_{v}^{N}$ will be defined in section 2. Instead of proving the above qualitative result, we in fact are able to obtain a quantitative bound for the discrepancy of the set of the rational points with bounded height.

In the classical theory of uniform distribution mod one, one can obtain an estimate for the discrepancy by using the Erdös-Turán inequality and estimating the resulting exponential sums. In our situation, we use Vaaler's approximation in [15] and certain orthonormal polynomials in place of the exponential function as it occurs in the classical case. First we obtain an analogue of the Erdös-Turán inequality for a general compact metric space in section 3. Then in section 4 we concentrate on the projective space $\mathbb{P}^{N-1}\left(k_{v}\right)$ and determine an explicit representation of the orthonormal polynomials in this case. If $v$ is an infinite place, the orthonormal polynomials can be expressed in terms of the usual Jacobi polynomials. In the final section, we estimate the summation of the orthonormal polynomials over the set of rational points with low height and then apply the Erdös-Turán inequality to prove our main result. 


\section{InVARIANt MEASURE ON $\mathbb{P}^{N-1}\left(k_{v}\right)$}

In this section we first introduce a $\sigma$-algebra on $\mathbb{P}^{N-1}\left(k_{v}\right)$ which contains all the Borel sets in $\mathbb{P}^{N-1}\left(k_{v}\right)$, and then, by using Haar measure on $k_{v}^{N}$, we define a positive measure on this $\sigma$-algebra. We will show that this measure is invariant under the group of isometries $\operatorname{ISO}\left(N, k_{v}\right)$.

As in [2], we select a Haar measure $\beta_{v}$ on the additive group of $k_{v}$ in the following manner:

(i) if $v \mid \infty$ and $k_{v} \cong \mathbb{R}$, then $\beta_{v}$ is the usual Lebesgue measure on $\mathbb{R}$,

(ii) if $v \mid \infty$ and $k_{v} \cong \mathbb{C}$, then $\beta_{v}$ is the usual Lebesgue measure on $\mathbb{C}$ multiplied by 2 ,

(iii) if $v \nmid \infty$, we require that $\beta_{v}\left(\boldsymbol{O}_{v}\right)=\left\|\mathcal{D}_{v}\right\|_{v}^{d_{v} / 2}$, where $\boldsymbol{O}_{v}:=\left\{\alpha \in k_{v}:\|\alpha\|_{v} \leq\right.$ $1\}$ and $\mathcal{D}_{v}$ is the local different of $k$ at $v$.

We denote the open and closed balls with center $\boldsymbol{\alpha}$ and radius $r$ with respect to $\|\cdot\|_{v}$ in $k_{v}^{N}-\{\mathbf{0}\}$ by $B(\boldsymbol{\alpha}, r)$ and $D(\boldsymbol{\alpha}, r)$ respectively. Also, $\mathbb{B}(\boldsymbol{\alpha}, r)$ and $\mathbb{D}(\boldsymbol{\alpha}, r)$ denote the projective open and closed balls with center $\boldsymbol{\alpha}$ and radius $r$ with respect to $\Delta_{v}(\boldsymbol{\alpha}, \boldsymbol{\beta})$ in $\mathbb{P}^{N-1}\left(k_{v}\right)$ respectively. We write $\beta_{v}^{N}$ for the $N$-fold product of measures $\beta_{v}$. From the way we have normalized $\beta_{v}$ we find that

$$
\beta_{v}^{N}(D(\mathbf{0}, 1))=r_{v}(N)^{-d_{v} N}
$$

at each infinite place $v$ of $k$. Since $\beta_{v}^{N}$ is invariant under translation, it follows that for any $\boldsymbol{\alpha} \in k_{v}^{N}$, we have

$$
\beta_{v}^{N}(B(\boldsymbol{\alpha}, 1))=\beta_{v}^{N}(D(\boldsymbol{\alpha}, 1))=r_{v}(N)^{-d_{v} N}
$$

at each infinite place $v$ of $k$. If $v \nmid \infty$, by (iii) above, we have for any $\boldsymbol{\alpha} \in k_{v}^{N}$,

$$
\beta_{v}^{N}(D(\boldsymbol{\alpha}, 1))=\left\|\mathcal{D}_{v}\right\|_{v}^{N d_{v} / 2} .
$$

For convenience, we denote $\beta_{v}^{N}(D(\mathbf{0}, 1))$ by $b_{v}(N)$. So, from (2.1) and (2.2),

$$
b_{v}(N)= \begin{cases}r_{v}(N)^{-d_{v} N} & \text { if } v \mid \infty, \\ \left\|\mathcal{D}_{v}\right\|_{v}^{N d_{v} / 2} & \text { if } v \nmid \infty .\end{cases}
$$

Let $\mathcal{B}$ be the $\sigma$-algebra of all Borel sets in $k_{v}^{N}-\{\mathbf{0}\}$. Define a collection $\mathcal{M}$ of subsets in $\mathbb{P}^{N-1}\left(k_{v}\right)$ by

$$
\mathcal{M}:=\left\{\mathbb{A} \subseteq \mathbb{P}^{N-1}\left(k_{v}\right): \phi^{-1}(\mathbb{A}) \in \mathcal{B}\right\} .
$$

Then it is clear that $\mathcal{M}$ is a $\sigma$-algebra in $\mathbb{P}^{N-1}\left(k_{v}\right)$ containing all the Borel sets in $\mathbb{P}^{N-1}\left(k_{v}\right)$. We now define a measure $\mu_{v}^{N}$ on $\mathbb{P}^{N-1}\left(k_{v}\right)$ as follows: if $\mathbb{A} \in \mathcal{M}$, then

$$
\mu_{v}^{N}(\mathbb{A}):=\frac{1}{b_{v}(N)} \beta_{v}^{N}\left(\phi^{-1}(\mathbb{A}) \cap D(\mathbf{0}, 1)\right) .
$$

It is straightforward to show that $\left(\mathbb{P}^{N-1}\left(k_{v}\right), \mathcal{M}, \mu_{v}^{N}\right)$ is a measure space and

$$
\mu_{v}^{N}\left(\mathbb{P}^{N-1}\left(k_{v}\right)\right)=1 \text {. }
$$

Since Haar measure on $k_{v}$ is unique up to a nonzero scalar multiple, $\mu_{v}^{N}$ is independent of our choice of $\beta_{v}$. It also follows from the definition (2.4) that if $F$ is a $\mu_{v}^{N}$-integrable function on $\mathbb{P}^{N-1}\left(k_{v}\right)$, then

$$
\int_{\mathbb{P}^{N-1}\left(k_{v}\right)} F(\boldsymbol{\beta}) d \mu_{v}^{N}(\boldsymbol{\beta})=\frac{1}{b_{v}(N)} \int_{D(\mathbf{0}, 1)} F(\phi(\boldsymbol{\alpha})) d \beta_{v}^{N}(\boldsymbol{\alpha}) .
$$


It is convenient to state the following formula for changing coordinates. This will be used frequently later. Let $f$ be $\beta_{v}^{N}$-integrable and $B$ an element in $\operatorname{GL}\left(N, k_{v}\right)$. Then we have

$$
\int_{k_{v}^{N}} F(\boldsymbol{\alpha}) d \beta_{v}^{N}(\boldsymbol{\alpha})=|\operatorname{det} B|_{v}^{d} \int_{k_{v}^{N}} F(B \boldsymbol{\alpha}) d \beta_{v}^{N}(\boldsymbol{\alpha})
$$

The next lemma shows that $\mu_{v}^{N}$ is invariant under the group of isometries $\operatorname{ISO}\left(N, k_{v}\right)$.

Lemma 2.1. Let $A$ be an element in $\operatorname{PGL}\left(N, k_{v}\right)$ and $F$ a non-negative $\mu_{v}^{N}$ integrable function on $\mathbb{P}^{N-1}\left(k_{v}\right)$. Then we have

$$
\begin{aligned}
\eta_{v}(A)^{-N d} & \int_{\mathbb{P}^{N-1}\left(k_{v}\right)} F(\boldsymbol{\beta}) d \mu_{v}^{N}(\boldsymbol{\beta}) \\
& \leq \int_{\mathbb{P}^{N-1}\left(k_{v}\right)} F(A \boldsymbol{\beta}) d \mu_{v}^{N}(\boldsymbol{\beta}) \leq \eta_{v}(A)^{N d} \int_{\mathbb{P}^{N-1}\left(k_{v}\right)} F(\boldsymbol{\beta}) d \mu_{v}^{N}(\boldsymbol{\beta})
\end{aligned}
$$

In particular, if $A$ belongs to $\operatorname{ISO}\left(N, k_{v}\right)$, then

$$
\int_{\mathbb{P}^{N-1}\left(k_{v}\right)} F(A \boldsymbol{\beta}) d \mu_{v}^{N}(\boldsymbol{\beta})=\int_{\mathbb{P}^{N-1}\left(k_{v}\right)} F(\boldsymbol{\beta}) d \mu_{v}^{N}(\boldsymbol{\beta})
$$

for any non-negative $\mu_{v}^{N}$-integrable function $F$ on $\mathbb{P}^{N-1}\left(k_{v}\right)$. Consequently, $\mu_{v}^{N}$ is invariant under $\operatorname{ISO}\left(N, k_{v}\right)$.

Proof. Suppose $F$ is a non-negative $\mu_{v}^{N}$-integrable function on $\mathbb{P}^{N-1}\left(k_{v}\right)$. By $(2.5)$ and (2.6), we have

$$
\begin{aligned}
& \int_{\mathbb{P}^{N-1}\left(k_{v}\right)} F(A \boldsymbol{\beta}) d \mu_{v}^{N}(\boldsymbol{\beta}) \\
= & \frac{1}{b_{v}(N)} \int_{D(\mathbf{0}, 1)} F(A \phi(\boldsymbol{\alpha})) d \beta_{v}^{N}(\boldsymbol{\alpha}) \\
= & \frac{1}{b_{v}(N)} \int_{k_{v}^{N}-\{\mathbf{0}\}} F(\phi(A \boldsymbol{\alpha})) \cdot \chi_{D(\mathbf{0}, 1)}\left(A^{-1} A \boldsymbol{\alpha}\right) d \beta_{v}^{N}(\boldsymbol{\alpha}) \\
= & \frac{1}{b_{v}(N)|\operatorname{det} A|_{v}^{d}} \int_{k_{v}^{N}-\{\mathbf{0}\}} F(\phi(\boldsymbol{\alpha})) \cdot \chi_{D(\mathbf{0}, 1)}\left(A^{-1} \boldsymbol{\alpha}\right) d \beta_{v}^{N}(\boldsymbol{\alpha}) \\
= & \frac{1}{b_{v}(N)|\operatorname{det} A|_{v}^{d}} \int_{0<\left|A^{-1} \boldsymbol{\alpha}\right|_{v} \leq 1} F(\phi(\boldsymbol{\alpha})) d \beta_{v}^{N}(\boldsymbol{\alpha}) .
\end{aligned}
$$

Since $\left|A^{-1} \boldsymbol{\alpha}\right|_{v} \leq\left|A^{-1}\right|_{v}|\boldsymbol{\alpha}|_{v}$, so by (1.7), (2.5) and (2.6), 


$$
\begin{aligned}
& \int_{\mathbb{P}^{N-1}\left(k_{v}\right)} F(A \boldsymbol{\beta}) d \mu_{v}^{N}(\boldsymbol{\beta}) \\
\geq & \frac{1}{b_{v}(N)|\operatorname{det} A|_{v}^{d}} \int_{0<|\boldsymbol{\alpha}|_{v} \leq\left|A^{-1}\right|_{v}^{-1}} F(\phi(\boldsymbol{\alpha})) d \beta_{v}^{N}(\boldsymbol{\alpha}) \\
= & \frac{1}{b_{v}(N)|\operatorname{det} A|_{v}^{d}\left|A^{-1}\right|_{v}^{N d}} \int_{k_{v}^{N}-\{\mathbf{0}\}} F(\phi(\boldsymbol{\alpha})) \cdot \chi_{D(\mathbf{0}, 1)}(\boldsymbol{\alpha}) d \beta_{v}^{N}(\boldsymbol{\alpha}) \\
= & \frac{\left|\operatorname{det} A^{-1}\right|_{v}^{d}}{\left|A^{-1}\right|_{v}^{N d}} \int_{\mathbb{P}^{N-1}\left(k_{v}\right)} F(\boldsymbol{\beta}) d \mu_{v}^{N}(\boldsymbol{\beta}) \\
\geq & \eta_{v}\left(A^{-1}\right)^{-N d} \int_{\mathbb{P}^{N-1}\left(k_{v}\right)} F(\boldsymbol{\beta}) d \mu_{v}^{N}(\boldsymbol{\beta}) \\
= & \eta_{v}(A)^{-N d} \int_{\mathbb{P}^{N-1}\left(k_{v}\right)} F(\boldsymbol{\beta}) d \mu_{v}^{N}(\boldsymbol{\beta}) .
\end{aligned}
$$

This proves the first inequality in (2.7). The second inequality follows from the first inequality if we replace $F$ by $F \circ A$ and $A$ by $A^{-1}$. Clearly the remaining assertions of Lemma 2.1 can be deduced directly from $(2.7)$ if $\eta_{v}(A)=1$.

With respect to this invariant measure, a sequence $\left\{\boldsymbol{\alpha}_{l}\right\}_{l=1}^{\infty}$ in $\mathbb{P}^{N-1}\left(k_{v}\right)$ is $\mu_{v^{-}}$ uniformly distributed if

$$
\lim _{L \longrightarrow \infty} \frac{1}{L} \sum_{l=1}^{L} f\left(\boldsymbol{\alpha}_{l}\right)=\int_{\mathbb{P}^{N-1}\left(k_{v}\right)} f(\boldsymbol{\alpha}) d \mu_{v}^{N}(\boldsymbol{\alpha}),
$$

for all real-valued continuous functions $f$ on $\mathbb{P}^{N-1}\left(k_{v}\right)$. The main result of this paper is the following theorem.

Theorem 2.2. The set $\left\{\boldsymbol{\beta} \in \mathbb{P}^{N-1}(k): H(\boldsymbol{\beta}) \leq H\right\}$ as $H \longrightarrow \infty$ is $\mu_{v}^{N}$-uniformly distributed.

We will also prove a quantitative result to be compared with the qualitative Theorem 2.2. In Theorem 5.11 at the end of this paper, we give an explicit estimation of the discrepancy for the set of all rational points with low height.

We finish this section by proving some formulae for the measures of the projective balls in $\mathbb{P}^{N-1}\left(k_{v}\right)$. These formulae are essential when we compute certain orthonormal polynomials for $\mathbb{P}^{N-1}\left(k_{v}\right)$ in section 4 . We first note that the measures of the projective balls are independent of their centers. For, if $\boldsymbol{\beta}_{1}$ and $\boldsymbol{\beta}_{2}$ belong to $\mathbb{P}^{N-1}\left(k_{v}\right)$ and $0 \leq r \leq 1$, since $\operatorname{ISO}\left(N, k_{v}\right)$ acts transitively on $\mathbb{P}^{N-1}\left(k_{v}\right)$ (e.g. Lemma 2.7 in [14]), there is an isometry $A$ such that $A \boldsymbol{\beta}_{2}=\boldsymbol{\beta}_{1}$. Hence by Lemma 2.1 ,

$$
\mu_{v}^{N}\left(\mathbb{B}\left(\boldsymbol{\beta}_{1}, r\right)\right)=\mu_{v}^{N}\left(\mathbb{B}\left(A \boldsymbol{\beta}_{2}, r\right)\right)=\mu_{v}^{N}\left(\mathbb{B}\left(\boldsymbol{\beta}_{2}, r\right)\right) .
$$

Similarly, we have $\mu_{v}^{N}\left(\mathbb{D}\left(\boldsymbol{\beta}_{1}, r\right)\right)=\mu_{v}^{N}\left(\mathbb{D}\left(\boldsymbol{\beta}_{2}, r\right)\right)$.

Next we divide our considerations into two cases : $v \mid \infty$ and $v \nmid \infty$. We first suppose that $v \mid \infty$. Of course, in this case, $\mu_{v}^{N}(\mathbb{B}(\boldsymbol{\beta}, r))=\mu_{v}^{N}(\mathbb{D}(\boldsymbol{\beta}, r))$. So, we only need to consider $\mathbb{D}(\boldsymbol{\beta}, r)$. Then we have the following lemma.

Lemma 2.3. Let $v \mid \infty$. For any $\boldsymbol{\beta}$ in $\mathbb{P}^{N-1}\left(k_{v}\right)$ and $0 \leq r \leq 1$, we have

$$
\mu_{v}^{N}(\mathbb{D}(\boldsymbol{\beta}, r))= \begin{cases}\frac{2}{c_{1}} \int_{0}^{r} \frac{x^{N-2}}{\sqrt{1-x^{2}}} d x & \text { if } k_{v} \cong \mathbb{R}, \\ r^{2(N-1)} & \text { if } k_{v} \cong \mathbb{C},\end{cases}
$$

where $c_{1}=c_{1}(N):=\pi^{\frac{1}{2}} \Gamma\left(\frac{N-1}{2}\right) / \Gamma\left(\frac{N}{2}\right)$. 
Proof. Since the measures of the projective balls are independent of their centers, without loss of generality, we can assume that $\boldsymbol{\beta}=\mathbf{e}_{1}:=[1,0, \cdots, 0]$. It is clear that (2.9) is true for $r=0$ and $r=1$, because

$$
\int_{0}^{1} \frac{x^{N-2}}{\sqrt{1-x^{2}}} d x=\frac{c_{1}}{2}
$$

Thus we assume $0<r<1$.

In view of (1.3),

$$
\begin{aligned}
& \phi^{-1}\left(\mathbb{D}\left(\mathbf{e}_{1}, r\right)\right) \cap D(\mathbf{0}, 1) \\
& =\left\{\left(\alpha_{0}, \cdots, \alpha_{N-1}\right) \in k_{v}^{N}-\{\mathbf{0}\}: \sum_{j=1}^{N-1}\left\|\alpha_{j}\right\|_{v}^{2} \leq \min \left\{\frac{r^{2}}{1-r^{2}}\left\|\alpha_{0}\right\|_{v}^{2}, 1-\left\|\alpha_{0}\right\|_{v}^{2}\right\}\right\} .
\end{aligned}
$$

Let $D_{N-1}(\boldsymbol{\alpha}, r)$ be the closed ball in $k_{v}^{N-1}$ with center $\boldsymbol{\alpha}$ and radius $r$. Hence,

$$
\begin{aligned}
& \mu_{v}^{N}\left(\mathbb{D}\left(\mathbf{e}_{1}, r\right)\right) \\
= & \frac{1}{b_{v}(N)}\left\{\int_{\left\|\alpha_{0}\right\|_{v} \leq\left(1-r^{2}\right)^{\frac{1}{2}}} \beta_{v}^{N-1}\left(D_{N-1}\left(\mathbf{0}, \frac{r\left\|\alpha_{0}\right\|_{v}}{\sqrt{1-r^{2}}}\right)\right) d \beta_{v}\left(\alpha_{0}\right)\right. \\
& \left.+\int_{\left(1-r^{2}\right)^{\frac{1}{2}}<\left\|\alpha_{0}\right\|_{v} \leq 1} \beta_{v}^{N-1}\left(D_{N-1}\left(\mathbf{0}, \sqrt{1-\left\|\alpha_{0}\right\|_{v}^{2}}\right)\right) d \beta_{v}\left(\alpha_{0}\right)\right\} .
\end{aligned}
$$

So, in view of $(2.1),(2.3)$ and (2.6), (2.10) becomes

$$
\begin{gathered}
\frac{r_{v}(N)^{N d_{v}}}{r_{v}(N-1)^{(N-1) d_{v}}}\left\{\left(\frac{r^{2}}{1-r^{2}}\right)^{\frac{N-1}{2} d_{v}} \int_{\left\|\alpha_{0}\right\|_{v} \leq\left(1-r^{2}\right)^{\frac{1}{2}}}\left\|\alpha_{0}\right\|_{v}^{(N-1) d_{v}} d \beta_{v}\left(\alpha_{0}\right)\right. \\
\left.\quad+\int_{\left(1-r^{2}\right)^{\frac{1}{2}}<\left\|\alpha_{0}\right\|_{v} \leq 1}\left(1-\left\|\alpha_{0}\right\|_{v}^{2}\right)^{\frac{N-1}{2} d_{v}} d \beta_{v}\left(\alpha_{0}\right)\right\} \\
:=\frac{r_{v}(N)^{N d_{v}}}{r_{v}(N-1)^{(N-1) d_{v}}\left\{\left(\frac{r^{2}}{1-r^{2}}\right)^{\frac{N-1}{2} d_{v}} I_{1}+I_{2}\right\} .}
\end{gathered}
$$

If $k_{v} \cong \mathbb{R}$, then $d_{v}=1$. Thus,

$$
I_{1}=2 \int_{0}^{\left(1-r^{2}\right)^{\frac{1}{2}}} t^{N-1} d t=\frac{2}{N}\left(1-r^{2}\right)^{\frac{N}{2}}
$$

and

$$
\begin{aligned}
I_{2} & =2 \int_{\left(1-r^{2}\right)^{\frac{1}{2}}}^{1}\left(1-t^{2}\right)^{\frac{N-1}{2}} d t=2 \int_{0}^{r} \frac{t^{N}}{\left(1-t^{2}\right)^{1 / 2}} d t \\
& =-\frac{2}{N} r^{N-1}\left(1-r^{2}\right)^{\frac{1}{2}}+\frac{2(N-1)}{N} \int_{0}^{r} \frac{t^{N-2}}{\left(1-t^{2}\right)^{1 / 2}} d t .
\end{aligned}
$$

Hence (2.9) follows from (2.10), (2.11) and the fact that

$$
\frac{N-1}{N} \cdot \frac{r_{v}(N)^{N}}{r_{v}(N-1)^{(N-1)}}=\frac{1}{c_{1}}
$$

for $k_{v} \cong \mathbb{R}$. 
If $k_{v} \cong \mathbb{C}$, then $d_{v}=2$. Then,

$$
\begin{aligned}
I_{1} & =2 \int_{x^{2}+y^{2} \leq 1-r^{2}}\left(x^{2}+y^{2}\right)^{N-1} d x d y \\
& =\frac{2 \pi}{N}\left(1-r^{2}\right)^{N}
\end{aligned}
$$

and

$$
\begin{aligned}
I_{2} & =2 \int_{1-r^{2}<x^{2}+y^{2} \leq 1}\left(1-\left(x^{2}+y^{2}\right)\right)^{N-1} d x d y \\
& =\frac{2 \pi}{N} r^{N} .
\end{aligned}
$$

Therefore, (2.9) follows from (2.10), (2.11) and the fact that

$$
\frac{r_{v}(N)^{2 N}}{r_{v}(N-1)^{2(N-1)}}=\frac{N}{2 \pi}
$$

for $k_{v} \cong \mathbb{C}$.

Next we consider the case $v \nmid \infty$. Let

$$
\boldsymbol{P}_{v}=\left\{\alpha \in k_{v}:\|\alpha\|_{v}<1\right\}
$$

be the maximal ideal in $\mathrm{mcl}_{v}$. Since $\|\cdot\|_{v}$ is discrete for $v \nmid \infty, \boldsymbol{P}_{v}$ is a principal ideal. If $\boldsymbol{P}_{v}=\left(\pi_{v}\right)$, then we say that $\pi_{v}$ is a prime element for $\|\cdot\|_{v}$. It is clear that

$$
\left\|\pi_{v}\right\|_{v}=\max \left\{\|\alpha\|_{v}: \alpha \in \boldsymbol{P}_{v}\right\} .
$$

As is well known (see for example Chapter 4 in [3]), for any $\alpha \in k_{v}^{*}:=k-\{0\}$, $\alpha=u \pi_{v}^{m}$ for some unit $u$ in $\boldsymbol{O}_{v}$ and some rational integer $m$. Thus $\|\alpha\|_{v}=\left\|\pi_{v}\right\|_{v}^{m}$, and therefore the multiplicative value group of $k_{v}$ is

$$
\left\{\|\alpha\|_{v}: \alpha \in k_{v}^{*}\right\}=\left\{\left\|\pi_{v}\right\|_{v}^{m}: m \in \mathbb{Z}\right\}
$$

It turns out that

$$
\left\{\Delta_{v}(\boldsymbol{\alpha}, \boldsymbol{\beta}): \boldsymbol{\alpha} \neq \boldsymbol{\beta}\right\}=\left\{\left\|\pi_{v}\right\|_{v}^{m}: m \geq 0\right\} .
$$

Thus, if we are considering the projective balls in $\mathbb{P}^{N-1}\left(k_{v}\right)$ for $v \nmid \infty$, we may assume that the radius has the form $\left\|\pi_{v}\right\|_{v}^{m}, m \geq 0$. Moreover, if we let $0<r \leq 1$ and assume that

$$
\left\|\pi_{v}\right\|_{v}^{m}<r \leq\left\|\pi_{v}\right\|_{v}^{m-1}
$$

for some $m \geq 1$, then

$$
\begin{aligned}
\mathbb{B}(\boldsymbol{\alpha}, r) & =\left\{\boldsymbol{\beta} \in \mathbb{P}^{N-1}\left(k_{v}\right): \Delta_{v}(\boldsymbol{\alpha}, \boldsymbol{\beta})<r\right\} \\
& =\left\{\boldsymbol{\beta} \in \mathbb{P}^{N-1}\left(k_{v}\right): \Delta_{v}(\boldsymbol{\alpha}, \boldsymbol{\beta}) \leq\left\|\pi_{v}\right\|_{v}^{m}\right\} \\
& =\mathbb{D}\left(\boldsymbol{\alpha},\left\|\pi_{v}\right\|_{v}^{m}\right) .
\end{aligned}
$$

This shows that we only need to consider $\mathbb{D}(\boldsymbol{\alpha}, r)$ with $r=\left\|\pi_{v}\right\|_{v}^{m}, m \geq 0$.

Lemma 2.4. Let $v \nmid \infty$, and let $\pi_{v}$ be a prime element for $\|\cdot\|_{v}$. For any $\boldsymbol{\beta} \in$ $\mathbb{P}^{N-1}\left(k_{v}\right), r=\left\|\pi_{v}\right\|_{v}^{m}$ and $m>0$, we have

$$
\mu_{v}^{N}(\mathbb{D}(\boldsymbol{\beta}, r))=r^{(N-1) d_{v}} \frac{1-\left\|\pi_{v}\right\|_{v}^{d_{v}}}{1-\left\|\pi_{v}\right\|_{v}^{N d_{v}}} .
$$


Proof. As before, we can assume that $\boldsymbol{\beta}=\mathbf{e}_{1}$ and $0<r<1$. In view of (1.3), we have

$$
\phi^{-1}\left(\mathbb{D}\left(\mathbf{e}_{1}, r\right)\right)=\left\{\left(\alpha_{0}, \cdots, \alpha_{N-1}\right) \in k_{v}^{N}-\{\mathbf{0}\}:\left\|\alpha_{j}\right\|_{v} \leq r\left\|\alpha_{0}\right\|_{v}, 1 \leq j \leq N-1\right\} .
$$

Thus, by (2.2) and (2.6)

$$
\begin{aligned}
\mu_{v}\left(\mathbb{D}\left(\mathbf{e}_{1}, r\right)\right) & =\frac{1}{b_{v}(N)} \beta_{v}^{N}\left(\phi^{-1}\left(\mathbb{D}\left(\mathbf{e}_{1}, r\right)\right) \cap D(\mathbf{0}, 1)\right) \\
& =\frac{1}{b_{v}(N)} \int_{D(\mathbf{0}, 1)} \chi_{\phi^{-1}\left(\mathbb{D}\left(\mathbf{e}_{1}, r\right)\right)}(\boldsymbol{\alpha}) d \beta_{v}^{N}(\boldsymbol{\alpha}) \\
& =\frac{1}{b_{v}(N)} \int_{\left\|\alpha_{0}\right\|_{v} \leq 1}\left\{\prod_{j=1}^{N-1} \int_{\left\|\alpha_{j}\right\|_{v} \leq r\left\|_{0}\right\|_{v}} d \beta_{v}\left(\alpha_{j}\right)\right\} d \beta_{v}\left(\alpha_{0}\right) \\
& =\frac{1}{b_{v}(N)} \int_{\left\|\alpha_{0}\right\|_{v} \leq 1} \beta_{v}\left(\left\{\alpha \in k_{v}:\|\alpha\|_{v} \leq r\left\|\alpha_{0}\right\|_{v}\right\}\right)^{N-1} d \beta_{v}\left(\alpha_{0}\right) \\
& =\frac{1}{b_{v}(N)} \int_{\left\|\alpha_{0}\right\|_{v} \leq 1}\left\{\left(r\left\|\alpha_{0}\right\|_{v}\right)^{d_{v}}\left\|\mathcal{D}_{v}\right\|_{v}^{d_{v} / 2}\right\}^{N-1} d \beta_{v}\left(\alpha_{0}\right) \\
& =\frac{1}{b_{v}(N)}\left(r\left\|\mathcal{D}_{v}\right\|_{v}^{1 / 2}\right)^{(N-1) d_{v}} \int_{\left\|\alpha_{0}\right\|_{v} \leq 1}\left\|\alpha_{0}\right\|_{v}^{(N-1) d_{v}} d \beta_{v}\left(\alpha_{0}\right) .
\end{aligned}
$$

To evaluate the last integral, we divide the set $\left\{\alpha_{0} \in k_{v}:\left\|\alpha_{0}\right\|_{v} \leq 1\right\}$ into a disjoint union of $\left\{\alpha_{0} \in k_{v}:\left\|\pi_{v}\right\|_{v}^{i+1}<\left\|\alpha_{0}\right\|_{v} \leq\left\|\pi_{v}\right\|_{v}^{i}\right\}$ for $i=0,1, \cdots$. So the last integral in (2.13) is equal to

$$
\begin{aligned}
& =\sum_{i=0}^{\infty}\left\|\pi_{v}\right\|_{v}^{i(N-1) d_{v}} \int_{\left\|\pi_{v}\right\|_{v}^{i+1}<\left\|\alpha_{0}\right\|_{v} \leq\left\|\pi_{v}\right\|_{v}^{i}} d \beta_{v}\left(\alpha_{0}\right) \\
& =\sum_{i=0}^{\infty}\left\|\pi_{v}\right\|_{v}^{i(N-1) d_{v}}\left\{\left\|\pi_{v}\right\|_{v}^{i d_{v}}\left\|\mathcal{D}_{v}\right\|_{v}^{d_{v} / 2}-\left\|\pi_{v}\right\|_{v}^{(i+1) d_{v}}\left\|\mathcal{D}_{v}\right\|_{v}^{d_{v} / 2}\right\} \\
& =\left(1-\left\|\pi_{v}\right\|_{v}^{d_{v}}\right)\left\|\mathcal{D}_{v}\right\|_{v}^{d_{v} / 2} \sum_{i=0}^{\infty}\left\|\pi_{v}\right\|_{v}^{i N d_{v}} \\
& =\frac{1-\left\|\pi_{v}\right\|_{v}^{d_{v}}}{1-\left\|\pi_{v}\right\|_{v}^{N d_{v}}}\left\|\mathcal{D}_{v}\right\|_{v}^{d_{v} / 2}
\end{aligned}
$$

Therefore, (2.12) follows from (2.3), (2.13) and (2.14).

\section{Uniform Distribution in a Compact Metric Space}

We will actually prove a quantitative form of Theorem 2.2 . We are able to give an explicit bound for the discrepancy of the set of rational points with low height for the infinite places, and then Theorem 2.2 follows as a corollary. Toward this end, we prove an analogue of the Erdös-Turán inequality for the projective space $\mathbb{P}^{N-1}\left(k_{v}\right)$. In fact we establish such a result for a general compact metric space.

In this section, we consider uniform distribution in a compact metric space. Let $X$ be a compact Hausdorff space and $\mu$ be a regular Borel probability measure in $X$. The sequence $\left\{x_{l}\right\}_{l=1}^{\infty}$ of elements in $X$ is said to be $\mu$-uniformly distributed in 
$X$ if

$$
\lim _{L \longrightarrow \infty} \frac{1}{L} \sum_{l=1}^{L} f\left(x_{l}\right)=\int_{X} f(x) d \mu
$$

for all real-valued continuous functions of $X$.

We further assume that $X$ is a metric space with metric $d$, and that the $\mu$ measure of both an open ball and a closed ball is independent of its center:

$$
\mu\left(B_{d}(x, r)\right)=\mu\left(B_{d}(y, r)\right) \quad \text { and } \quad \mu\left(D_{d}(x, r)\right)=\mu\left(D_{d}(y, r)\right)
$$

for any $x, y$ in $X$ and $r \geq 0$, where $B_{d}(x, r)$ and $D_{d}(x, r)$ are the open and closed balls in $X$ with center $x$ and radius $r$ respectively. Then by approximating continuous functions by a finite linear combination of characteristic functions of Borel sets in $X$, it follows that a sequence $\left\{x_{l}\right\}_{l=1}^{\infty}$ is $\mu$-uniformly distributed if and only if

$$
\lim _{L \longrightarrow \infty} \frac{1}{L} \sum_{l=1}^{L} \chi_{D_{d}(x, r)}\left(x_{l}\right)=\mu\left(D_{d}(x, r)\right)
$$

for any $x$ in $X$ and $r>0$, where $\chi_{E}$ is the characteristic function of the set $E$. Similarly, a sequence of $\left\{x_{l}\right\}_{l=1}^{\infty}$ is $\mu$-uniformly distributed if and only if

$$
\lim _{L \longrightarrow \infty} \frac{1}{L} \sum_{l=1}^{L} \chi_{B_{d}(x, r)}\left(x_{l}\right)=\mu\left(B_{d}(x, r)\right)
$$

for any $x$ in $X$ and $r>0$.

It is sometimes more convenient to consider normalized functions when we study their Fourier series. We define $f: \mathbb{R} \longrightarrow \mathbb{C}$ to be normalized if

$$
\lim _{h \longrightarrow 0^{+}} \frac{1}{2}\{f(x+h)+f(x-h)\}=f(x) .
$$

We also define the normalized characteristic function of a ball with center $x$ and radius $r$ by

$$
\chi_{(x, r)}(y):= \begin{cases}1 & \text { if } d(x, y)<r \\ \frac{1}{2} & \text { if } d(x, y)=r \\ 0 & \text { if } d(x, y)>r .\end{cases}
$$

Thus, $\chi_{(x, r)}(y)=\frac{1}{2}\left\{\chi_{B_{d}(x, r)}(y)+\chi_{D_{d}(x, r)}(y)\right\}$, and hence, in view of (3.2) and (3.3), we have

Lemma 3.1. The sequence $\left\{x_{l}\right\}_{l=1}^{\infty}$ is $\mu$-uniformly distributed if and only if

$$
\lim _{L \longrightarrow \infty} \frac{1}{L} \sum_{l=1}^{L} \chi_{(x, r)}\left(x_{l}\right)=\int_{X} \chi_{(x, r)}(x) d \mu .
$$

In the remainder of this section, we are going to prove an analogue of Weyl's criterion and the Erdös-Turán inequality for a general compact metric space. It is well known (e.g. Theorem 1.3 of Chapter 4 in [8]) that if $X$ is also a group, then we can obtain results analogous to Weyl's criterion by using the group characters. However, in our case, we are going to employ orthonormal polynomials. 
Let $\omega$ be a regular Borel probability measure on $[-1,1]$. Define the inner product by

$$
\langle f, g\rangle:=\int_{-1}^{1} f(t) \bar{g}(t) d \omega
$$

for any $f$ and $g$ in $L^{1}(\omega)$. $\left\{Q_{n}^{\omega}(x)\right\}_{n=1}^{\infty}$ is said to be the set of orthonormal polynomials with respect to $\omega$ if $Q_{n}^{\omega}(x)$ is polynomial of degree $n$ with positive leading coefficient and satisfies

$$
\left\langle Q_{m}^{\omega}, Q_{n}^{\omega}\right\rangle=\delta_{m n}:= \begin{cases}1 & \text { if } m=n, \\ 0 & \text { if } m \neq n .\end{cases}
$$

Suppose the set of orthonormal polynomials with respect to $\omega$ exists. Let $T_{m}(x)$ be the $m$-th Chebysheff polynomial, so that $T_{m}(\cos \theta)=\cos m \theta$. Since every polynomial can be expressed as a linear combination of the $Q_{n}^{\omega}$, we write $T_{m}$ as

$$
T_{m}(x)=\sum_{n=0}^{m} t_{n}^{m} Q_{n}^{\omega}(x),
$$

where $t_{n}^{m}:=\left\langle T_{m}, Q_{n}^{\omega}\right\rangle$ for $m, n \geq 0$. In view of (3.6), if $m<n$, then

$$
t_{n}^{m}=0 \text {. }
$$

In [15], J. Vaaler showed how to construct a trigonometric polynomial approximation to a function by using Beurling's extremal function. He also used this approximation to give an improved version of the classical Erdös-Turán inequality. In this section, we will employ Vaaler's approximation to prove the analogous ErdösTurán inequality. In [7], P. Grabner used a similar idea to obtain the Erdös-Turán inequality for the $N$-dimensional sphere.

We first recall some definitions used in Vaaler's approximation. Let $M$ be a positive integer. We write $e(x):=e^{2 \pi i x}$ and define

$$
j_{M}(x):=\sum_{m=-M}^{M} \widehat{J}_{M+1}(m) e(m x),
$$

where

$$
\widehat{J}_{M+1}(m):= \begin{cases}1 & \text { if } m=0 \\ \frac{\pi m}{M+1}\left(1-\frac{|m|}{M+1}\right) \cot \frac{\pi m}{M+1}+\frac{|m|}{M+1} & \text { if } 0<|m| \leq M, \\ 0 & \text { otherwise }\end{cases}
$$

and the periodic Fejer kernel

$$
k_{M}(x):=\sum_{m=-M}^{M} \widehat{K}_{M+1}(m) e(m x)
$$

where

$$
\widehat{K}_{M+1}(m):= \begin{cases}1-\frac{|m|}{M+1} & \text { if }|m| \leq M \\ 0 & \text { otherwise }\end{cases}
$$

It is easy to prove that

$$
k_{M}(x)=\frac{1}{M+1}\left(\frac{\sin \pi(M+1) x}{\sin \pi x}\right)^{2} .
$$


We now suppose that $g: \mathbb{R} \longrightarrow \mathbb{C}$ has period 1 and bounded variation on each closed interval of length 1. We also assume that $g$ satisfies the normalizing condition (3.4). Let $V_{g}(x)$ be the total variation of $g$ on $\left[-\frac{1}{2}, x\right]$. We write $\left(d V_{g}\right) * k_{M}(x)$ for the convolution

$$
\left(d V_{g}\right) * k_{M}(x):=\int_{-\frac{1}{2}}^{\frac{1}{2}} k_{M}(x-t) d V_{g}(t) .
$$

Then the trigonometric polynomial

$$
g * j_{M}(x):=\int_{-\frac{1}{2}}^{\frac{1}{2}} g(t) j_{M}(x-t) d t
$$

will give a good approximation to $g$. In view of Theorem 19 in [15], we have

$$
\left|g(x)-g * j_{M}(x)\right| \leq \frac{1}{2 M+2}\left(d V_{g}\right) * k_{M}(x)
$$

for any real number $x$. Inequality (3.14) is essential in our proof of the ErdösTurán type inequality. We are going to use (3.14) to approximate the normalized characteristic function.

Let

$$
\hat{g}(m):=\int_{-\frac{1}{2}}^{\frac{1}{2}} g(t) e(-m t) d t \quad \text { and } \quad \widehat{d V_{g}}(m):=\int_{-\frac{1}{2}}^{\frac{1}{2}} e(-m t) d V_{g}(t)
$$

be the $m$-th Fourier coefficients of $g$ and $d V_{g}$ respectively. If $g$ is an even function, then

$$
\hat{g}(m)=\hat{g}(-m) \quad \text { and } \quad \widehat{d V_{g}}(m)=\widehat{d V_{g}}(-m)
$$

Now we suppose that $g$ is an even function. Since $g * j_{M}$ is an even trigonometric polynomial, it can be written as a finite linear combination of $T_{m}(\cos 2 \pi x)$. Hence, in view of $(3.7), g * j_{M}$ can also be written as a linear combination of $Q_{n}^{\omega}(\cos 2 \pi x)$. From (3.9) and (3.13), for any real number $x$,

$$
\begin{aligned}
g * j_{M}(x) & =\int_{-\frac{1}{2}}^{\frac{1}{2}} g(t) j_{M}(x-t) d t \\
& =\sum_{m=-M}^{M} \hat{J}_{M+1}(m) e(m x) \int_{-\frac{1}{2}}^{\frac{1}{2}} g(t) e(-m t) d t \\
& =\sum_{m=-M}^{M} \hat{J}_{M+1}(m) \hat{g}(m) e(m x) .
\end{aligned}
$$


Using (3.7), (3.8) and (3.15), we get

$$
\begin{aligned}
g * j_{M}(x) & =\hat{g}(0)+2 \sum_{m=1}^{M} \hat{J}_{M+1}(m) \hat{g}(m) \cos 2 \pi m x \\
& =\hat{g}(0)+2 \sum_{m=1}^{M} \hat{J}_{M+1}(m) \hat{g}(m) T_{m}(\cos 2 \pi x) \\
& =\hat{g}(0)+2 \sum_{m=1}^{M} \hat{J}_{M+1}(m) \hat{g}(m) \sum_{n=0}^{m} t_{n}^{m} Q_{n}^{\omega}(\cos 2 \pi x) \\
& =\hat{g}(0)+\sum_{n=0}^{M} A_{n}^{M}(g) Q_{n}^{\omega}(\cos 2 \pi x)
\end{aligned}
$$

where

$$
A_{n}^{M}(g):=2 \sum_{m=1}^{M} \hat{J}_{M+1}(m) \hat{g}(m) t_{n}^{m}
$$

for $n \geq 0$. Similarly, we can also write $\left(d V_{g}\right) * k_{M}(x)$ as a linear combination of $Q_{n}^{\omega}(\cos 2 \pi x)$. Using (3.7), (3.8), (3.10) and (3.12), for any real number $x$, we have

$$
\left(d V_{g}\right) * k_{M}(x)=V_{g}\left(\frac{1}{2}\right)+\sum_{n=0}^{M} B_{n}^{M}(g) Q_{n}^{\omega}(\cos 2 \pi x),
$$

where

$$
B_{n}^{M}(g):=2 \sum_{m=1}^{M} \hat{K}_{M+1}(m) \widehat{d V_{g}}(m) t_{n}^{m}
$$

for any $n \geq 0$.

Lemma 3.2. Suppose $\omega$ is a regular Borel probability measure in $[-1,1]$ and the set $\left\{Q_{n}^{\omega}\right\}_{n=1}^{\infty}$ of orthonormal polynomials, with respect to $\omega$ exists. Let $f$ be normalized, $\omega$-measurable and of bounded variation on $[-1,1]$. Let $\left\{x_{l}\right\}_{l=1}^{\infty}$ be a sequence of real numbers in $[-1,1]$. Suppose

$$
g(x)=f(\cos 2 \pi x) .
$$

Then for any $L, M \geq 1$, we have

$$
\begin{aligned}
& \left|\frac{1}{L} \sum_{l=1}^{L} f\left(x_{l}\right)-\int_{-1}^{1} f(t) d \omega\right| \\
& \quad \leq \frac{\left|V_{g}\left(\frac{1}{2}\right)+B_{0}^{M}(g)\right|}{M+1}+\sum_{n=1}^{M}\left\{\left|A_{n}^{M}(g)\right|+\frac{\left|B_{n}^{M}(g)\right|}{2(M+1)}\right\}\left|\frac{1}{L} \sum_{l=1}^{L} Q_{n}^{\omega}\left(x_{l}\right)\right|,
\end{aligned}
$$

where $A_{n}^{M}(g)$ and $B_{n}^{M}(g)$ are defined in (3.17) and (3.19) respectively. 
Proof. Lemma 3.2 is a direct consequence of (3.14). For any $L, M \geq 1$,

$$
\begin{aligned}
\left|\frac{1}{L} \sum_{l=1}^{L} f\left(x_{l}\right)-\int_{-1}^{1} f(t) d \omega\right| \\
=\left|\frac{1}{L} \sum_{l=1}^{L} g\left(\frac{\cos ^{-1} x_{l}}{2 \pi}\right)-\int_{-1}^{1} g\left(\frac{\cos ^{-1} t}{2 \pi}\right) d \omega\right| \\
\leq\left|\frac{1}{L} \sum_{l=1}^{L} g * j_{M}\left(\frac{\cos ^{-1} x_{l}}{2 \pi}\right)-\int_{-1}^{1} g * j_{M}\left(\frac{\cos ^{-1} t}{2 \pi}\right) d \omega\right| \\
\quad+\frac{1}{2 M+2}\left\{\frac{1}{L} \sum_{l=1}^{L}\left(d V_{g}\right) * k_{M}\left(\frac{\cos ^{-1} x_{l}}{2 \pi}\right)+\int_{-1}^{1}\left(d V_{g}\right) * k_{M}\left(\frac{\cos ^{-1} t}{2 \pi}\right) d \omega\right\},
\end{aligned}
$$

by (3.14). In view of (3.6) and (3.16), the first term in the right hand side of (3.21) is equal to

$$
\begin{gathered}
\left|\sum_{n=0}^{M} A_{n}^{M}(g)\left\{\frac{1}{L} \sum_{l=1}^{L} Q_{n}^{\omega}\left(x_{l}\right)-\int_{-1}^{1} Q_{n}^{\omega}(t) d \omega\right\}\right| \\
=\left|\sum_{n=1}^{M} A_{n}^{M}(g) \frac{1}{L} \sum_{l=1}^{L} Q_{n}^{\omega}\left(x_{l}\right)\right|
\end{gathered}
$$

Similarly, from (3.6) and (3.18), the second term in the right hand side of (3.21) is equal to

$$
\frac{1}{2 M+2}\left\{2\left(V_{g}\left(\frac{1}{2}\right)+B_{0}^{M}(g)\right)+\sum_{n=1}^{M} B_{n}^{M}(g) \frac{1}{L} \sum_{l=1}^{L} Q_{n}^{\omega}\left(x_{l}\right)\right\} .
$$

Therefore, (3.20) follows from (3.21), (3.22) and (3.23).

We return to consideration of a general compact metric space. Let $(X, d, \mu)$ be an infinite compact metric space with regular Borel measure $\mu$ such that

(a) $\mu(X)=1$,

(b) $0 \leq d(x, y) \leq 1$ for any $x, y$ in $X$,

(c) the $\mu$-measures of balls in $X$ are independent of their centers.

Fixing $x_{0}$ in $X$, we define, for any open interval $U$ in $[-1,1]$,

$$
\omega(U)=\frac{1}{2} \int_{U} d\left\{\operatorname{sgn}(t) \mu\left(D_{d}\left(x_{0},|t|\right)\right)\right\}
$$

where $\operatorname{sgn}(t)$ is $+1,0$ and -1 according as $t \geq 0, t=0$, and $t<0$ respectively and the above integral is a Riemann-Stieltjes integral on $[-1,1]$. In view of (c) above, $\omega$ is independent of the choice of $x_{0}$ in $X$. From (3.24), we see that if $f$ is integrable, then

$$
\begin{aligned}
\int_{-1}^{1} f(t) d \omega & =\frac{1}{2} \int_{0}^{1} f(t) d \mu\left(D_{d}\left(x_{0}, t\right)\right)-\frac{1}{2} \int_{-1}^{0} f(t) d \mu\left(D_{d}\left(x_{0},|t|\right)\right) \\
& =\frac{1}{2} \int_{0}^{1}\{f(t)+f(-t)\} d \mu\left(D_{d}\left(x_{0}, t\right)\right)
\end{aligned}
$$


In particular, we have $\omega([-1,1])=\mu\left(D_{d}\left(x_{0}, 1\right)\right)=\mu(X)=1$, and if $f$ is an odd function then $\int_{-1}^{1} f(t) d \omega=0$. We suppose that the set $\left\{Q_{n}^{\omega}\right\}_{n=1}^{\infty}$ of orthonormal polynomials, with respect to $\omega$ exists. We write $Q_{n}^{\omega}:=Q_{e}+Q_{o}$, where $Q_{e}$ and $Q_{o}$ are the polynomials consisting of the even and odd terms in $Q_{n}^{\omega}$ respectively. If $n$ is even, then $\operatorname{deg}\left(Q_{o}\right)<n$, and in view of (3.6) and (3.25), we have

$$
0=\left\langle Q_{n}^{\omega}, Q_{o}\right\rangle=\left\langle Q_{e}+Q_{o}, Q_{o}\right\rangle=\left\langle Q_{o}, Q_{o}\right\rangle .
$$

This implies that $Q_{o}=0$ and hence $Q_{n}^{\omega}$ is even. Similarly, if $n$ is odd, then $Q_{n}^{\omega}$ is odd. Also, since $T_{m}(-x)=(-1)^{m} T_{m}(x)$, we have

$$
\begin{aligned}
t_{n}^{m} & =\int_{-1}^{1} T_{m}(x) Q_{n}^{\omega}(x) d \omega \\
& =\frac{1}{2} \int_{0}^{1}\left\{T_{m}(x) Q_{n}^{\omega}(x)+T_{m}(-x) Q_{n}^{\omega}(-x)\right\} d \mu\left(D_{d}\left(x_{0}, t\right)\right) \\
& =\frac{1+(-1)^{m+n}}{2} \int_{0}^{1} T_{m}(x) Q_{n}^{\omega}(x) d \mu\left(D_{d}\left(x_{0}, t\right)\right)
\end{aligned}
$$

for any $m, n \geq 0$. We conclude that if $m+n$ is odd, then

$$
t_{n}^{m}=0 \text {. }
$$

Next we apply Lemma 3.2 to the normalized characteristic function. Let $0<$ $r \leq 1$ and

$$
f(t):=\frac{1}{2}\left\{\chi_{(-r, r)}(t)+\chi_{[-r, r]}(t)\right\}
$$

Then we have by (3.5)

$$
\begin{aligned}
\chi_{(y, r)}(x) & =\frac{1}{2}\left\{\chi_{(-r, r)}(d(x, y))+\chi_{[-r, r]}(d(x, y))\right\} \\
& =f(d(x, y)) .
\end{aligned}
$$

If $\mu\left(D_{d}\left(x_{0}, t\right)\right)$ is continuous at $r$ as a function of $t$ on $[0,1]$, then the characteristic functions $\chi_{(-r, r)}$ and $\chi_{[-r, r]}$ are Riemann-Stieltjes integrable on $[-1,1]$, and

$$
\begin{aligned}
\int_{X} \chi_{(y, r)}(x) d \mu & =\int_{X} \frac{1}{2}\left\{\chi_{B_{d}(y, r)}(x)+\chi_{D_{d}(y, r)}(x)\right\} d \mu \\
& =\int_{-1}^{1} \frac{1}{2}\left\{\chi_{(-r, r)}(t)+\chi_{[-r, r]}(t)\right\} d \omega \\
& =\int_{-1}^{1} f(t) d \omega .
\end{aligned}
$$

Thus, in view of Lemma 3.2, we have

$$
\begin{aligned}
& \left|\frac{1}{L} \sum_{l=1}^{L} \boldsymbol{\chi}_{(y, r)}\left(x_{l}\right)-\int_{X} \chi_{(y, r)}(x) d \mu\right| \\
& \quad=\left|\frac{1}{L} \sum_{l=1}^{L} f\left(d\left(x_{l}, y\right)\right)-\int_{-1}^{1} f(t) d \omega\right| \\
& \quad \leq \frac{\left|V_{g}\left(\frac{1}{2}\right)+B_{0}^{M}(g)\right|}{M+1}+\sum_{n=1}^{M}\left\{\left|A_{n}^{M}(g)\right|+\frac{\left|B_{n}^{M}(g)\right|}{2(M+1)}\right\}\left|\frac{1}{L} \sum_{l=1}^{L} Q_{n}^{\omega}\left(d\left(x_{l}, y\right)\right)\right|
\end{aligned}
$$


where $g(x)=\frac{1}{2}\left\{\chi_{(-r, r)}+\chi_{[-r, r]}\right\}(\cos 2 \pi x)$. We should remark here that when $r=1, f$ does not satisfy the normalizing condition (3.4). However, (3.14) still holds for $g(x)$ by computing both sides of (3.14) directly. Hence (3.27) still holds for $r=1$.

Since $g(x)$ is an even function and $g\left(\frac{1}{2}-x\right)=g(x)$, it follows that if $m$ is odd, then $\hat{g}(m)=\widehat{d V_{g}}(m)=0$. Hence, in view of (3.17), (3.19) and (3.26), $A_{n}^{M}(g)$ and $B_{n}^{M}(g)$ are zero when $n$ is odd. Therefore, we have the following theorem.

Theorem 3.3 (Erdös-Turán Inequality). Suppose $(X, d, \mu)$ is an infinite compact metric space with regular Borel measure $\mu$ satisfying conditions (a), (b) and (c), and $\left\{x_{l}\right\}_{l=1}^{\infty}$ is a sequence in $X$. Let $\omega(x)$ be defined as in (3.24) and suppose the set $\left\{Q_{n}^{\omega}(x)\right\}_{n=1}^{\infty}$ of orthonormal polynomials, with respect to $\omega$ exists. For any $y$ in $X, 0<r \leq 1$ and $L, M \geq 1$, if $\mu\left(D_{d}\left(x_{0}, t\right)\right)$ is continuous at $r$, we have

$$
\begin{aligned}
& \left|\frac{1}{L} \sum_{l=1}^{L} \chi_{(y, r)}\left(x_{l}\right)-\int_{X} \chi_{(y, r)}(x) d \mu\right| \\
& \quad \leq \frac{\left|V_{g}\left(\frac{1}{2}\right)+B_{0}^{M}(g)\right|}{M+1}+\sum_{n=1}^{[M / 2]}\left\{\left|A_{2 n}^{M}(g)\right|+\frac{\left|B_{2 n}^{M}(g)\right|}{2(M+1)}\right\}\left|\frac{1}{L} \sum_{l=1}^{L} Q_{2 n}^{\omega}\left(d\left(x_{l}, y\right)\right)\right|,
\end{aligned}
$$

where $g(x)=\frac{1}{2}\left\{\chi_{(-r, r)}+\chi_{[-r, r]}\right\}(\cos 2 \pi x) ; A_{n}^{M}(g)$ and $B_{n}^{M}(g)$ are defined in $(3.17)$ and (3.19) respectively.

In order to deduce Weyl's criterion from the above theorem, we must have

$$
\lim _{M \longrightarrow \infty} \frac{\left|V_{g}\left(\frac{1}{2}\right)+B_{0}^{M}(g)\right|}{M+1}=0 .
$$

The following lemma gives an explicit bound for this.

Lemma 3.4. Under the same hypotheses as in Theorem 3.3, for sufficiently small $\epsilon>0$, we have

$$
\begin{aligned}
& \left|V_{g}\left(\frac{1}{2}\right)+B_{0}^{M}(g)\right| \\
\leq & 4\left(1+\frac{1}{\epsilon}+M\left\{\mu\left(D_{d}\left(x_{0}, r+\epsilon\right)\right)-\mu\left(D_{d}\left(x_{0}, r-\epsilon\right)\right)\right\}\right) .
\end{aligned}
$$

Proof. For $0<r<1$, we let $r=\cos 2 \pi \theta$ for some $\theta$ in $\left(0, \frac{1}{4}\right)$ and assume $0<\epsilon<\theta$. Then $V_{g}(x)$ is a step function on $\left[-\frac{1}{2}, \frac{1}{2}\right]$ having jump 1 at four points $\pm \theta$ and 
$\pm\left(\frac{1}{2}-\theta\right)$ only. In view of (3.6), (3.12), (3.18) and (3.25), we have

$$
\begin{aligned}
& V_{g}\left(\frac{1}{2}\right)+B_{0}^{M}(g) \\
= & \int_{-1}^{1}\left(d V_{g}\right) * k_{M}\left(\frac{\cos ^{-1} t}{2 \pi}\right) d \omega \\
= & \int_{-1}^{1}\left\{\int_{-\frac{1}{2}}^{\frac{1}{2}} k_{M}\left(\frac{\cos ^{-1} t}{2 \pi}-x\right) d V_{g}(x)\right\} d \omega \\
= & \int_{0}^{1}\left\{k_{M}\left(\frac{\cos ^{-1} t}{2 \pi}+\frac{1}{2}-\theta\right)+k_{M}\left(\frac{\cos ^{-1} t}{2 \pi}+\theta\right)\right. \\
& \left.+k_{M}\left(\frac{\cos ^{-1} t}{2 \pi}-\theta\right)+k_{M}\left(\frac{\cos ^{-1} t}{2 \pi}-\frac{1}{2}+\theta\right)\right\} d \mu\left(D_{d}\left(x_{0}, t\right)\right) .
\end{aligned}
$$

On the other hand, in view of (3.11), we have

$$
k_{M}(\phi) \leq 1+\min \left\{M,(2\|\phi\|)^{-1}\right\}
$$

for $M \geq 1$ and $\phi \in \mathbb{R}$. Here $\|x\|:=\min (\{x\}, 1-\{x\})$ is the distance from $x$ to the nearest integer.

Since both $\left\|\frac{\cos ^{-1} t}{2 \pi}+\frac{1}{2}-\theta\right\|$ and $\left\|\frac{\cos ^{-1} t}{2 \pi}+\theta\right\|$ are greater than $\theta$ for any $t$ in $[0,1]$, the contribution of the first two integrands in the right hand side of (3.30) is less than

$$
2 \int_{0}^{1}\left(1+(2 \theta)^{-1}\right) d \mu\left(D_{d}\left(x_{0}, t\right)\right)=2\left(1+(2 \theta)^{-1}\right)
$$

by (3.31). Next we consider

$$
\begin{aligned}
& \int_{0}^{1} k_{M}\left(\frac{\cos ^{-1} t}{2 \pi}-\theta\right) d \mu\left(D_{d}\left(x_{0}, t\right)\right) \\
= & \left\{\int_{0}^{r-\epsilon}+\int_{r-\epsilon}^{r+\epsilon}+\int_{r+\epsilon}^{1}\right\} k_{M}\left(\frac{\cos ^{-1} t}{2 \pi}-\theta\right) d \mu\left(D_{d}\left(x_{0}, t\right)\right) .
\end{aligned}
$$

For $t \in[0, r-\epsilon]$ or $t \in[r+\epsilon, 1]$, we have

$$
\left\|\frac{\cos ^{-1} t}{2 \pi}-\theta\right\|=\left|\frac{\cos ^{-1} t}{2 \pi}-\theta\right|=\left|\frac{\cos ^{-1} t}{2 \pi}-\frac{\cos ^{-1} r}{2 \pi}\right| \geq \frac{\epsilon}{2 \pi} .
$$

It follows from (3.31) that

$$
\begin{aligned}
& \int_{0}^{1} k_{M}\left(\frac{\cos ^{-1} t}{2 \pi}-\theta\right) d \mu\left(D_{d}\left(x_{0}, t\right)\right) \\
\leq & \left\{\int_{0}^{r-\epsilon}+\int_{r+\epsilon}^{1}\right\}\left(1+\frac{\pi}{\epsilon}\right) d \mu\left(D_{d}\left(x_{0}, t\right)\right)+\int_{r-\epsilon}^{r+\epsilon}(1+M) d \mu\left(D_{d}\left(x_{0}, t\right)\right) \\
\leq & 1+\frac{\pi}{\epsilon}+(1+M)\left\{\mu\left(D_{d}\left(x_{0}, r+\epsilon\right)\right)-\mu\left(D_{d}\left(x_{0}, r-\epsilon\right)\right)\right\} .
\end{aligned}
$$

Similarly, we have

$$
\begin{aligned}
& \int_{-1}^{1} k_{M}\left(\frac{\cos ^{-1} t}{2 \pi}-\frac{1}{2}+\theta\right) d \omega(t) \\
\leq & 1+\frac{\pi}{\epsilon}+(1+M)\left\{\mu\left(D_{d}\left(x_{0}, r+\epsilon\right)\right)-\mu\left(D_{d}\left(x_{0}, r-\epsilon\right)\right)\right\} .
\end{aligned}
$$


Therefore, from (3.30), (3.32), (3.34) and (3.34)

$$
\begin{aligned}
& V_{g}\left(\frac{1}{2}\right)+B_{0}^{M}(g) \\
\leq & 2\left(1+(2 \theta)^{-1}\right)+2\left(1+\frac{\pi}{\epsilon}\right)+2(1+M)\left\{\mu\left(D_{d}\left(x_{0}, r+\epsilon\right)\right)-\mu\left(D_{d}\left(x_{0}, r-\epsilon\right)\right)\right\} \\
\leq & 4\left(1+\frac{1}{\epsilon}+M\left\{\mu\left(D_{d}\left(x_{0}, r+\epsilon\right)\right)-\mu\left(D_{d}\left(x_{0}, r-\epsilon\right)\right)\right\}\right) .
\end{aligned}
$$

This proves (3.29) for $0<r<1$. The case $r=1$ can be proved in a similar manner.

Theorem 3.5 (Weyl's Criterion). Suppose $(X, d, \mu), \omega(x)$ and $Q_{n}^{\omega}(x)$ satisfy the conditions in Theorem 3.3, and that $\mu\left(D_{d}\left(x_{0}, t\right)\right)$ is continuous on $[0,1]$. Then the sequence $\left(x_{l}\right)_{l=1}^{\infty}$ is $\mu$-uniformly distributed if and only if

$$
\lim _{L \longrightarrow \infty} \frac{1}{L} \sum_{l=1}^{L} Q_{2 n}^{\omega}\left(d\left(x_{l}, y\right)\right)=0
$$

for any $y \in X$ and $n \geq 1$.

Proof. Suppose (3.35) holds for any $y \in X$ and $n \geq 1$. The result follows from Lemma 3.1, Theorem 3.3 and Lemma 3.4 by letting $\epsilon=M^{-1 / 2}$ and $M, L \longrightarrow \infty$. Conversely, suppose the sequence $\left\{x_{l}\right\}_{l=1}^{\infty}$ is $\mu$-uniformly distributed. Then (3.35) follows from (3.1) and the fact that

$$
\int_{X} Q_{2 n}^{\omega}(d(x, y)) d \mu=\int_{-1}^{1} Q_{2 n}^{\omega}(t) d \omega=0,
$$

for any $y \in X$ and $n \geq 1$.

\section{Uniform Distribution IN $\mathbb{P}^{N-1}\left(k_{v}\right)$}

In this section, we concentrate on the projective spaces over number fields again and compute the orthonormal polynomials for this case. In view of Lemmas 2.3, 2.4 and $\S 2.2$ in [11], the set of orthonormal polynomials exists. We denote the set of orthonormal polynomials for $\mathbb{P}^{N-1}\left(k_{v}\right)$ by $\left\{Q_{n}^{N, v}(x)\right\}_{n=1}^{\infty}$.

We first consider the case $v \mid \infty$. According to Lemma 2.3, we should divide our consideration into two cases: $k_{v} \cong \mathbb{R}$ and $k_{v} \cong \mathbb{C}$.

For $k_{v} \cong \mathbb{R}$, from (2.9), we have

$$
d \mu_{v}^{N}(\mathbb{D}(\boldsymbol{\beta}, t))=\frac{2}{c_{1}} \cdot \frac{t^{N-2}}{\sqrt{1-t^{2}}} d t .
$$

For any $\alpha, \beta>-1$ and $n \geq 0$, let $P_{n}^{(\alpha, \beta)}(x)$ be the Jacobi polynomials and for $\lambda>-\frac{1}{2}$ and $n \geq 0$, let $C_{n}^{\lambda}(x)$ be the ultraspherical polynomials (cf. (4.3.3) and (4.7.1) in [11]). Note that $C_{n}^{\lambda}(x)$ is the Jacobi polynomial with $\alpha=\beta=\lambda-\frac{1}{2}$ and suitable normalization. In particular, we have $C_{n}^{0}(\cos \theta)=\frac{2}{n} \cos n \theta$ and $C_{n}^{1}(\cos \theta)=$ $\frac{\sin (n+1) \theta}{\sin \theta}$. Like cosine and sine functions, Jacobi polynomials satisfy the addition 
theorem ((4.10.20) in [11]), namely,

$$
\begin{aligned}
P_{n}^{(\alpha, \beta)} & \left(2\left|\cos \theta_{1} \cos \theta_{2}+r e^{i \phi} \sin \theta_{1} \sin \theta_{2}\right|^{2}-1\right) \\
= & \sum_{m=0}^{n} \sum_{l=0}^{m} a_{m, n, l}^{(\alpha, \beta)}\left(\sin \theta_{1} \sin \theta_{2}\right)^{m+l}\left(\cos \theta_{1} \cos \theta_{2}\right)^{m-l} P_{n-m}^{(\alpha+m+l, \beta+m-l)}\left(\cos 2 \theta_{1}\right) \\
& \times P_{n-m}^{(\alpha+m+l, \beta+m-l)}\left(\cos 2 \theta_{2}\right) P_{l}^{(\alpha-\beta-1, \beta+m-l)} \\
& \times\left(2 r^{2}-1\right) r^{m-l} \frac{\beta+m-l}{\beta} C_{m-l}^{\beta}(\cos \phi),
\end{aligned}
$$

where

$$
\begin{gathered}
a_{m, n, l}^{(\alpha, \beta)}=\frac{(m+l+\alpha) \Gamma(n+m+\alpha+\beta+1) \Gamma(m+\alpha)}{\Gamma(n+\alpha+\beta+1) \Gamma(n+l+\alpha+1)} \\
\times \frac{\Gamma(\beta+1) \Gamma(n+\beta+1) \Gamma(n-m+1)}{\Gamma(m+\beta+1) \Gamma(n-l+\beta+1)}
\end{gathered}
$$

and the limit relation

$$
\lim _{\beta \longrightarrow 0} \frac{\beta+n}{\beta} C_{n}^{\beta}(\cos \phi)= \begin{cases}2 \cos n \phi, & n=1,2, \cdots, \\ 1, & n=0,\end{cases}
$$

is used when $\beta=0$. In particular, for the ultraspherical polynomials, we have

$$
\begin{aligned}
& C_{n}^{\lambda}(\cos \theta \cos \phi+\sin \theta \sin \phi \cos \psi) \\
& =C_{n}^{\lambda}(\cos \theta) C_{n}^{\lambda}(\cos \phi)+\sum_{j=1}^{n} b_{j, n}^{\lambda}(\sin \theta)^{j} C_{n-j}^{\lambda+j}(\cos \theta) \\
& \quad \times(\sin \phi)^{j} C_{n-j}^{\lambda+j}(\cos \phi) \frac{2 \lambda+2 j-1}{2 \lambda-1} C_{j}^{\lambda-\frac{1}{2}}(\cos \psi),
\end{aligned}
$$

where

$$
b_{j, n}^{\lambda}=\frac{\Gamma(2 \lambda) 2^{2 j}(\Gamma(j+\lambda))^{2} \Gamma(n-j+1)}{(\Gamma(\lambda))^{2} \Gamma(n+j+2 \lambda)},
$$

subject to the same limit relation above. It is easy to show that

$$
a_{m, n, l}^{(\alpha, 0)} \ll\left(\frac{n+m+\alpha}{n-m+1}\right)^{m-l} \quad \text { and } \quad b_{j, n}^{\lambda} \ll_{\lambda} 2^{2 j}
$$

for $\alpha \geq 0$ and $2 \lambda \in \mathbb{N}$. Also in view of (7.32.2) and (7.33.1) in [11], if $\alpha, \beta \geq 0$ and $\lambda \geq \frac{1}{2}$, then we have

$$
\left|P_{n}^{(\alpha, \beta)}(x)\right| \leq(e n)^{\max (\alpha, \beta)}, \quad\left|P_{n}^{(\alpha, \beta)^{\prime}}(x)\right| \leq \frac{\alpha+\beta+n+1}{2}(\text { en })^{\max (\alpha, \beta)+1}
$$

and

$$
\left|C_{n}^{\lambda}(x)\right| \leq(e n)^{2 \lambda-1}, \quad\left|C_{n}^{\lambda^{\prime}}(x)\right| \leq 2 \lambda(e n)^{2 \lambda+1}
$$

for any $|x| \leq 1$ and $n \geq 1$. These estimations will be frequently used in the next section.

The next lemma shows that the orthonormal polynomials for the case $k_{v} \cong \mathbb{R}$ can be expressed in terms of the ultraspherical polynomials. 
Lemma 4.1. Suppose $v \mid \infty$ and $k_{v} \cong \mathbb{R}$. Then for $n \geq 0$

$$
Q_{n}^{N, v}(x)= \begin{cases}\alpha_{n}^{N} C_{n}^{\frac{N-2}{2}}\left(\sqrt{1-x^{2}}\right) & \text { if } n \text { is even, } \\ \alpha_{n}^{N} x C_{n-1}^{\frac{N}{2}}\left(\sqrt{1-x^{2}}\right) & \text { if } n \text { is odd, }\end{cases}
$$

where $\alpha_{0}^{N}=1$ and for $n \geq 1$

$$
\alpha_{n}^{N}:= \begin{cases}(-1)^{\frac{n-1}{2}}\left\{\frac{N-1}{N+2 n-2}\left(\begin{array}{c}
N+n-2 \\
n-1
\end{array}\right)\right\}^{-\frac{1}{2}} & \text { if } n \text { is odd, } \\
(-1)^{\frac{n}{2}} n 2^{-\frac{1}{2}} & \text { if } n \text { is even and } N=2, \\
(-1)^{\frac{n}{2}}\left\{\frac{N-2}{N+2 n-2}\left(\begin{array}{c}
N+n-3 \\
n
\end{array}\right)\right\}^{-\frac{1}{2}} & \text { if } n \text { is even and } N \geq 3 .\end{cases}
$$

Proof. In view of the uniqueness of the orthonormal polynomials, it suffices to show that the polynomials in (4.6) satisfy condition (3.6), because $Q_{n}^{N, v}(x)$ is a polynomial of degree $n$ with positive leading coefficient. First of all, since $Q_{n}^{N, v}(x)$ is odd and even according as $n$ is odd and even, so $\left\langle Q_{2 m}^{N, v}(x), Q_{2 n+1}^{N, v}(x)\right\rangle=0$ for any $m, n \geq 0$ by (3.25). Now we consider

$$
\begin{aligned}
& \left\langle Q_{2 m}^{N, v}(x), Q_{2 n}^{N, v}(x)\right\rangle \\
= & \alpha_{2 m}^{N} \alpha_{2 n}^{N} \int_{-1}^{1} C_{2 m}^{\frac{N-2}{2}}\left(\sqrt{1-x^{2}}\right) C_{2 n}^{\frac{N-2}{2}}\left(\sqrt{1-x^{2}}\right) d \omega \\
= & \frac{2 \alpha_{2 m}^{N} \alpha_{2 n}^{N}}{c_{1}} \int_{0}^{1} C_{2 m}^{\frac{N-2}{2}}\left(\sqrt{1-x^{2}}\right) C_{2 n}^{\frac{N-2}{2}}\left(\sqrt{1-x^{2}}\right) \frac{x^{N-2}}{\sqrt{1-x^{2}}} d x \\
= & \frac{\alpha_{2 m}^{N} \alpha_{2 n}^{N}}{c_{1}} \int_{-1}^{1} C_{2 m}^{\frac{N-2}{2}}(x) C_{2 n}^{\frac{N-2}{2}}(x)\left(1-x^{2}\right)^{\frac{N-3}{2}} d x \\
= & \delta_{m n},
\end{aligned}
$$

by (3.25), (4.1), (4.3.3) and (4.7.1) in [11]. Similarly, we can prove that

$$
\left\langle Q_{2 m+1}^{N, v}(x), Q_{2 n+1}^{N, v}(x)\right\rangle=\delta_{m n} .
$$

This completes the proof of the lemma.

For $k_{v} \cong \mathbb{C}$, from $(2.9)$, we have

$$
d \mu_{v}^{N}(\mathbb{D}(\boldsymbol{\beta}, t))=2(N-1) t^{2 N-3} d t .
$$

In a manner similar to the case $k_{v} \cong \mathbb{R}, Q_{n}^{N, v}(x)$ can be expressed in terms of the Jacobi polynomials.

Lemma 4.2. Suppose $v \mid \infty$ and $k_{v} \cong \mathbb{C}$. Then for any $m \geq 0$

$$
Q_{n}^{N, v}(x)= \begin{cases}\beta_{n}^{N} P_{m}^{(0, N-2)}\left(2 x^{2}-1\right) & \text { if } n=2 m, \\ \beta_{n}^{N} x P_{m}^{(0, N-1)}\left(2 x^{2}-1\right) & \text { if } n=2 m+1,\end{cases}
$$

where $\beta_{n}^{N}=\left(\frac{N+n-1}{N-1}\right)^{\frac{1}{2}}$.

Proof. The proof is similar to that of Lemma 4.1.

When $v$ is a finite place, the orthonormal polynomials in this case are more complicated and we are not able to represent them in terms of familiar polynomials. Since the explicit formula for these polynomials doesn't contribute to our proof of the main theorem, we just state the following recursive relation for $Q_{n}^{N, v}(x)$ without proof. A proof can be found in [4]. 
Let

$$
\alpha_{m}^{n}:=1-\left\|\pi_{v}\right\|_{v}^{2 n(n-1)+m+((n+1) N-n) d_{v}} \quad \text { and } \quad \beta_{m}:=1-\left\|\pi_{v}\right\|_{v}^{m+(N-1) d_{v}}
$$

for any $m \geq 0$ and $n \geq-1$.

Lemma 4.3. For $n \geq 2$, we have

$$
Q_{n}^{N, v}(x)=a_{n-1}\left\{x Q_{n-1}^{N, v}(x)-\frac{1}{a_{n-2}} Q_{n-2}^{N, v}(x)\right\}
$$

and $Q_{0}^{N, v}(x) \equiv 1, Q_{1}^{N, v}(x) \equiv\left(\frac{\alpha_{0}^{0} \beta_{2}}{\beta_{0} \alpha_{2}^{0}}\right)^{\frac{1}{2}} x$. Here

$$
a_{2 n}=\left\{\frac{\alpha_{4 n}^{n} \alpha_{6 n-4}^{n-1} \beta_{4 n+2} \beta_{4 n}}{\alpha_{4 n-4}^{n-1} \alpha_{6 n+2}^{n} \beta_{2 n}^{2}\left\|\pi_{v}\right\|_{v}^{2 n}}\right\}^{\frac{1}{2}}
$$

and

$$
a_{2 n+1}=\left\{\frac{\alpha_{6 n+2}^{n} \alpha_{4 n}^{n} \beta_{4 n+4} \beta_{4 n+2}}{\alpha_{6 n-4}^{n-1} \alpha_{4 n+4}^{n+1}\left(1-\left\|\pi_{v}\right\|_{v}^{2 n+2}\right)^{2}\left\|\pi_{v}\right\|_{v}^{2 n+(N-1) d_{v}}}\right\}^{\frac{1}{2}},
$$

for $n \geq 0$.

We conclude this section by proving the Weyl criterion for $\mathbb{P}^{N-1}\left(k_{v}\right)$.

Theorem 4.4 (Weyl's Criterion). Let $\left\{\boldsymbol{\alpha}_{l}\right\}_{l=1}^{\infty}$ be a sequence in $\mathbb{P}^{N-1}\left(k_{v}\right)$. Then $\left\{\boldsymbol{\alpha}_{l}\right\}_{l=1}^{\infty}$ is $\mu_{v}^{N}$-uniformly distributed if and only if

$$
\lim _{L \longrightarrow \infty} \frac{1}{L} \sum_{l=1}^{L} Q_{2 n}^{N, v}\left(\Delta_{v}\left(\boldsymbol{\alpha}_{l}, \boldsymbol{\beta}\right)\right)=0
$$

for any $\boldsymbol{\beta}$ in $\mathbb{P}^{N-1}\left(k_{v}\right)$ and $n \geq 1$.

Proof. For $v \mid \infty$, since $\mu_{v}^{N}(\mathbb{D}(\boldsymbol{\beta}, t))$ is continuous on [0,1], (4.8) follows from Theorem 3.5. For $v \nmid \infty$, we recall that

$$
\left\{\Delta_{v}(\boldsymbol{\alpha}, \boldsymbol{\beta}): \boldsymbol{\alpha} \neq \boldsymbol{\beta}\right\}=\left\{\left\|\pi_{v}\right\|_{v}^{m}: m \geq 0\right\} .
$$

Thus for any $\boldsymbol{\beta} \in \mathbb{P}^{N-1}\left(k_{v}\right)$ and $m>0$, we have

$$
\chi_{\mathbb{D}\left(\boldsymbol{\beta},\left\|\pi_{v}\right\|_{v}^{m}\right)}(\boldsymbol{\alpha})=\chi_{\left(\boldsymbol{\beta}, \frac{\left\|\pi_{v}\right\|_{v}^{m-1}}{2}\left(1+\left\|\pi_{v}\right\|_{v}\right)\right)}(\boldsymbol{\alpha}) .
$$

Since $\mu_{v}^{N}(\mathbb{D}(\boldsymbol{\beta}, t))$ is constant on $\left(\left\|\pi_{v}\right\|_{v}^{m},\left\|\pi_{v}\right\|_{v}^{m-1}\right)$, if we take

$$
r=\frac{\left\|\pi_{v}\right\|_{v}^{m-1}}{2}\left(1+\left\|\pi_{v}\right\|_{v}\right) \text { and } \epsilon=\frac{\left\|\pi_{v}\right\|_{v}^{m-1}}{4}\left(1-\left\|\pi_{v}\right\|_{v}\right)
$$

in (3.29), then

$$
\frac{\left|V_{g}\left(\frac{1}{2}\right)+B_{0}^{M}(g)\right|}{M+1} \leq \frac{4\left(1+\frac{1}{\epsilon}\right)}{M+1} \leq \frac{32}{\left(1-\left\|\pi_{v}\right\|_{v}\right)\left\|\pi_{v}\right\|_{v}^{m-1}(M+1)} \longrightarrow 0
$$

as $M \longrightarrow \infty$. Hence, by (3.28), for any $\boldsymbol{\beta} \in \mathbb{P}^{N-1}\left(k_{v}\right)$ and $m, n \geq 1$, (4.8) implies

$$
\lim _{L \longrightarrow \infty} \frac{1}{L} \sum_{l=1}^{L} \chi_{\mathbb{D}\left(\boldsymbol{\beta},\left\|\pi_{v}\right\|_{v}^{m}\right)}\left(\boldsymbol{\alpha}_{l}\right)=\mu\left(\mathbb{D}\left(\boldsymbol{\beta},\left\|\pi_{v}\right\|_{v}^{m}\right)\right) .
$$

Hence, from (3.1) and (3.2), $\left\{\boldsymbol{\alpha}_{l}\right\}_{l=1}^{\infty}$ is $\mu_{v}^{N}$-uniformly distributed if and only if (4.8) holds. This completes the proof of Theorem 4.4. 


\section{Rational Points with Low Height}

We now come to the proof of Theorem 2.2. For $v \mid \infty$, in view of Weyl's criterion, we are going to establish the following result.

Theorem 5.1. Suppose $v \mid \infty$. Let $H \geq 1$, and let $n$ be a positive integer. Then for any $\boldsymbol{\beta}$ in $\mathbb{P}^{N-1}\left(k_{v}\right)$, we have

$$
\sum_{\substack{\boldsymbol{\alpha} \in \mathbb{P}^{N-1}(k) \\ H(\boldsymbol{\alpha}) \leq H}} Q_{2 n}^{N, v}\left(\Delta_{v}(\boldsymbol{\alpha}, \boldsymbol{\beta})\right)=O\left(n^{9(N-1) n}\left(H^{N d-1}+H \log H\right)\right),
$$

where the implicit constant depends only on $k$ and $N$.

Although using a slightly different definition of height, S. Schanuel in [10] (also see Theorem 5.3 in [6] and Theorem 1 in [13]) proved the following asymptotic formula for the number of rational points with low height:

$$
\sum_{\substack{\boldsymbol{\alpha} \in \mathbb{P}^{N-1}(k) \\ H(\boldsymbol{\alpha}) \leq H}} 1=c_{2} H^{N d}+O\left(H^{N d-1}+H \log H\right),
$$

where

$$
c_{2}=c_{2}(N, k):=\frac{h R 2^{N r_{2}} N^{r_{1}+r_{2}-1}(V(N))^{r_{1}}(V(2 N))^{r_{2}}}{w\left\|\Delta_{k}\right\|^{\frac{N}{2}} \zeta_{k}(N)} .
$$

Here $h$ is the class number, $r_{1}$ and $r_{2}$ are the number of real and complex embeddings respectively from $k$ into $\mathbb{C}, R$ is the regulator, $w$ is the number of roots of unity of $k, \zeta_{k}$ is the Dedekind zeta function of $k$ and $V(l)$ is the $l$-dimensional volume of the unit ball in $\mathbb{R}^{l}$. Then Theorem 2.2 follows from Theorem 4.4, (5.2) and Theorem 5.1.

Let

$$
\mathfrak{O}_{k}:=\left\{\alpha \in k:\|\alpha\|_{v} \leq 1 \text { for all } v, v \nmid \infty\right\}
$$

be the ring of integers in $k$. If $v$ is a finite place of $k$, then we define

$$
\mathfrak{P}_{v}:=\left\{\alpha \in \mathfrak{O}_{k}:\|\alpha\|_{v}<1\right\} .
$$

Then $\left\{\mathfrak{P}_{v}: v \nmid \infty\right\}$ is the set of all prime ideals in $\mathfrak{O}_{k}$. In view of the fundamental theorem of ideals in $k$, every non-zero fractional ideal $\mathfrak{B}$ in $k$ can be written uniquely as a product of prime ideals such that

$$
\mathfrak{B}=\prod_{v \nmid \infty} \mathfrak{P}_{v}^{m_{v}}
$$

for $m_{v} \in \mathbb{Z}$ and $m_{v}=0$ for almost all but finitely many $v$. Furthermore, we have

$$
\mathfrak{B}=\left\{\alpha \in k:\|\alpha\|_{v} \leq\left\|\pi_{v}\right\|_{v}^{m_{v}} \text { for all } v, v \nmid \infty\right\} .
$$

If we denote the norm $N\left(\mathfrak{P}_{v}\right)$ of $\mathfrak{P}_{v}$ by $q_{v}$, then

$$
N(\mathfrak{B})=\prod_{v \nmid \infty} N\left(\mathfrak{P}_{v}\right)^{m_{v}}=\prod_{v \nmid \infty} q_{v}^{m_{v}}
$$

and $q_{v}=\left\|\pi_{v}\right\|_{v}^{-d_{v}}$.

Let $\alpha \longrightarrow \alpha^{(i)}(1 \leq i \leq d)$ denote the embeddings from $k$ into $\mathbb{C}$, ordered so that the first $r_{1}$ are real and $\alpha^{\left(i+r_{2}\right)}=\overline{\alpha^{(i)}}$ for $r_{1}+1 \leq i \leq r_{1}+r_{2}$, where $\bar{\alpha}$ is the 
complex conjugate of $\alpha$ and $d=r_{1}+2 r_{2}$. If $\boldsymbol{\alpha}=\left(\alpha_{0}, \cdots, \alpha_{N-1}\right)^{t}$ is an element in $k^{N}$, then we let

$$
\boldsymbol{\alpha}^{(i)}=\left(\alpha_{0}^{(i)}, \cdots, \alpha_{N-1}^{(i)}\right)^{t}
$$

where $\boldsymbol{\alpha}^{t}$ means the transpose of $\boldsymbol{\alpha}$. Hence if $v$ is an infinite place of $k$, then $\|\boldsymbol{\alpha}\|_{v}=\left\|\boldsymbol{\alpha}^{(i)}\right\|$ for some $1 \leq i \leq r_{1}+r_{2}$, where $\|\cdot\|$ is the usual Euclidean norm over $\mathbb{C}$.

Given a non-zero element $\boldsymbol{\alpha}$ in $k^{N}$, we let $\langle\boldsymbol{\alpha}\rangle$ be the fractional ideal in $k$ generated by its components $\alpha_{0}, \cdots, \alpha_{N-1}$. For each finite place $v$, we let $\|\boldsymbol{\alpha}\|_{v}=\left\|\pi_{v}\right\|_{v}^{m_{v}}$ for some $m_{v} \in \mathbb{Z}$. Then

$$
\langle\boldsymbol{\alpha}\rangle=\left\{\alpha \in k:\|\alpha\|_{v} \leq\left\|\pi_{v}\right\|_{v}^{m_{v}} \text { for all } v, v \nmid \infty\right\}=\prod_{v \nmid \infty} \mathfrak{P}_{v}^{m_{v}} .
$$

It follows that

$$
\begin{aligned}
N(\langle\boldsymbol{\alpha}\rangle) & =\prod_{v \nmid \infty}\left\|\pi_{v}\right\|_{v}^{-m_{v} d_{v}} \\
& =\prod_{v \nmid \infty}\|\boldsymbol{\alpha}\|_{v}^{-d_{v}}
\end{aligned}
$$

and hence

$$
H(\boldsymbol{\alpha})^{d}=N(\langle\boldsymbol{\alpha}\rangle)^{-1} \prod_{i=1}^{r_{1}+r_{2}}\left\|\boldsymbol{\alpha}^{(i)}\right\|^{e_{i}}
$$

where

$$
e_{i}:= \begin{cases}1 & \text { if } 1 \leq i \leq r_{1} \\ 2 & \text { if } r_{1}+1 \leq i \leq r_{1}+r_{2}\end{cases}
$$

It will be convenient to write

$$
H_{\infty}(\boldsymbol{\alpha}):=\prod_{i=1}^{r_{1}+r_{2}}\left\|\boldsymbol{\alpha}^{(i)}\right\|^{e_{i}}
$$

so that

$$
H(\boldsymbol{\alpha})^{d}=N(\langle\boldsymbol{\alpha}\rangle)^{-1} H_{\infty}(\boldsymbol{\alpha}) .
$$

Let $\mu$ be the Möbius function on ideals in $\mathfrak{O}_{k}$.

Lemma 5.2. Let $F: \mathbb{P}^{N-1}(k) \longrightarrow \mathbb{C}$ be a bounded function and $\|F(\boldsymbol{\alpha})\| \leq M$ for any $\boldsymbol{\alpha}$ in $\mathbb{P}^{N-1}(k)$. Then for any $H \geq 1$ we have

$$
\begin{aligned}
& \sum_{\substack{\boldsymbol{\alpha} \in \mathbb{P}^{N-1}(k) \\
H(\boldsymbol{\alpha}) \leq H}} F(\boldsymbol{\alpha})=\sum_{\substack{\mathfrak{C} \subseteq \mathfrak{O}_{k} \\
N(\mathfrak{C}) \leq H^{d}}} \mu(\mathfrak{C}) \sum_{\substack{\mathfrak{B} \subseteq \mathfrak{O}_{k} \\
N(\mathfrak{B}) \leq H^{d} / N(\mathfrak{C})}} \\
& \times \sum_{\substack{\boldsymbol{\alpha} \in\left(\mathfrak{B}^{-1}\right)^{N-1} \\
H_{\infty}(\boldsymbol{\alpha}, 1) \leq H^{d} / N(\mathfrak{B C})}} F([\boldsymbol{\alpha}, 1])+O\left(M H^{(N-1) d}\right),
\end{aligned}
$$

where $\mathfrak{C} \subseteq \mathfrak{O}_{k}$ means that the summation runs over all integral ideals in $\mathfrak{O}_{k}$ and the implicit constant depends only on $k$.

Proof. For any non-zero $\boldsymbol{\alpha}=\left(\alpha_{0}, \cdots, \alpha_{N-2}\right)^{t} \in k^{N-1}$, we define

$$
\mathfrak{T}(\boldsymbol{\alpha}):=\left\{a \in k: a \boldsymbol{\alpha} \in \mathfrak{O}_{k}^{N-1}\right\} .
$$


Then $\mathfrak{T}(\boldsymbol{\alpha})$ is a fractional ideal in $k$. Let

$$
\mathfrak{T}^{*}(\boldsymbol{\alpha}):=\mathfrak{T}(\boldsymbol{\alpha}) \cap \mathfrak{O}_{k} .
$$

So, $\mathfrak{T}^{*}(\boldsymbol{\alpha})=\langle(\boldsymbol{\alpha}, 1)\rangle^{-1}$. Hence from (5.2), (5.4) and (5.5), we have

$$
\begin{aligned}
& \sum_{\substack{\boldsymbol{\alpha} \in \mathbb{P}^{N-1}(k) \\
H(\boldsymbol{\alpha}) \leq H}} F(\boldsymbol{\alpha})=\sum_{\substack{[\boldsymbol{\alpha}, 1] \in \mathbb{P}^{N-1}(k) \\
H(\boldsymbol{\alpha}, 1) \leq H}} F([\boldsymbol{\alpha}, 1])+O\left(M H^{(N-1) d}\right) \\
& =\sum_{\substack{\boldsymbol{\alpha} \in k^{N-1} \\
N\left(\mathfrak{T}^{*}(\boldsymbol{\alpha})\right) H_{\infty}(\boldsymbol{\alpha}, 1) \leq H^{d}}} F([\boldsymbol{\alpha}, 1])+O\left(M H^{(N-1) d}\right) .
\end{aligned}
$$

For any fractional ideal $\mathfrak{U}$ in $k$, we define

$$
\lambda(\mathfrak{U}, H):=\sum_{\substack{\boldsymbol{\alpha} \in k^{N-1} \\ \mathfrak{T}(\boldsymbol{\alpha}) \supseteq \mathfrak{U} \\ H_{\infty}(\boldsymbol{\alpha}, 1) \leq H / N(\mathfrak{U})}} F([\boldsymbol{\alpha}, 1])
$$

and

$$
\bar{\lambda}(\mathfrak{U}, H):=\sum_{\substack{\boldsymbol{\alpha} \in k^{N-1} \\ \mathfrak{T}^{*}(\boldsymbol{\alpha})=\mathfrak{U} \\ H_{\infty}(\boldsymbol{\alpha}, 1) \leq H / N(\mathfrak{U})}} F([\boldsymbol{\alpha}, 1]) .
$$

Using an argument similar to that used to prove Lemma 1 in [13], we have

$$
\bar{\lambda}(\mathfrak{U}, H)=\sum_{\mathfrak{C} \mid \mathfrak{U}} \mu(\mathfrak{C}) \lambda\left(\mathfrak{U} \mathfrak{C}^{-1}, H / N(\mathfrak{C})\right) .
$$

Note that if $\mathfrak{U} \nsubseteq \mathfrak{O}_{k}$, then in view of $(5.9), \bar{\lambda}(\mathfrak{U}, H)=0$. So, by (5.10),

$$
\begin{aligned}
& \sum_{\substack{\boldsymbol{\alpha} \in k^{N-1} \\
N\left(\mathfrak{T}^{*}(\boldsymbol{\alpha})\right) H_{\infty}(\boldsymbol{\alpha}, 1) \leq H^{d}}} F([\boldsymbol{\alpha}, 1]) \\
&= \sum_{\substack{\mathfrak{U} \subseteq \mathfrak{O}_{k} \\
N(\mathfrak{U}) \leq H^{d}}} \sum_{\substack{\boldsymbol{\alpha} \in k^{N-1}, \mathfrak{T}^{*}(\boldsymbol{\alpha})=\mathfrak{U} \\
H_{\infty}(\boldsymbol{\alpha}, 1) \leq H^{d} / N(\mathfrak{U})}} F([\boldsymbol{\alpha}, 1]) \\
&= \sum_{\substack{\mathfrak{U} \subseteq \mathfrak{O}_{k} \\
N(\mathfrak{U}) \leq H^{d}}} \bar{\lambda}\left(\mathfrak{U}, H^{d}\right) \\
&= \sum_{\substack{\mathfrak{U} \subseteq \mathfrak{O}_{k} \\
N(\mathfrak{U}) \leq H^{d}}} \sum_{\substack{\mathfrak{C} \mid \mathfrak{U} \\
\mathfrak{C} \subseteq \mathfrak{O}_{k} \\
N(\mathfrak{C}) \leq H^{d}}} \mu(\mathfrak{C}) \lambda\left(\mathfrak{U} \mathfrak{C}^{-1}, H^{d} / N(\mathfrak{C})\right) \\
& \sum_{\substack{\mathfrak{B} \subseteq \mathfrak{O}_{k} \\
N(\mathfrak{B}) \leq H^{d} / N(\mathfrak{C})}} \lambda\left(\mathfrak{B}, H^{d} / N(\mathfrak{C})\right)
\end{aligned}
$$

where $\mathfrak{U}=\mathfrak{B C}$.

Now suppose $\mathfrak{B}$ is a fractional ideal in $k$. Then we claim that

$$
\boldsymbol{\alpha} \in\left(\mathfrak{B}^{-1}\right)^{N-1} \quad \text { if and only if } \quad \mathfrak{B} \subseteq \mathfrak{T}(\boldsymbol{\alpha}) .
$$

For, if $\boldsymbol{\alpha}=\left(\alpha_{0}, \cdots, \alpha_{N-2}\right)^{t} \in\left(\mathfrak{B}^{-1}\right)^{N-1}$, then

$$
\alpha_{j} \mathfrak{B} \subseteq \mathfrak{O}_{k}
$$


for all $0 \leq j \leq N-2$. So, $\mathfrak{B} \subseteq \mathfrak{T}(\boldsymbol{\alpha})$. Conversely, if $\mathfrak{B} \subseteq \mathfrak{T}(\boldsymbol{\alpha})$, then for any $b \in \mathfrak{B}$, we have $b \boldsymbol{\alpha} \in \mathfrak{O}_{k}^{N-1}$ and hence $b \alpha_{j} \in \mathfrak{O}_{k}$ for all $j=0, \cdots, N-2$. Therefore $\alpha_{j} \mathfrak{B} \subseteq \mathfrak{O}_{k}$ for all $j=0, \cdots, N-2$, and this implies that $\boldsymbol{\alpha} \in\left(\mathfrak{B}^{-1}\right)^{N-1}$.

Finally, in view of (5.8) and (5.12), we get

$$
\lambda\left(\mathfrak{B}, H^{d} / N(\mathfrak{C})\right)=\sum_{\substack{\boldsymbol{\alpha} \in\left(\mathfrak{B}^{-1}\right)^{N-1} \\ H_{\infty}(\boldsymbol{\alpha}, 1) \leq H^{d} / N(\mathfrak{B} \mathfrak{C})}} F([\boldsymbol{\alpha}, 1]) .
$$

Therefore, (5.6) follows from (5.7), (5.11) and (5.13).

Lemma 5.2 suggests that we should investigate the sum

$$
S^{l}(\mathfrak{B}, X, F):=\sum_{\substack{\boldsymbol{\alpha} \in\left(\mathfrak{B}^{-1}\right)^{l-1} \\ H_{\infty}(\boldsymbol{\alpha}, 1) \leq X}} F(\boldsymbol{\alpha})
$$

for any $X \geq 1, l \geq 2$ and integral ideal $\mathfrak{B}$ in $\mathfrak{O}_{k}$. For $\alpha \in \mathbb{C}$, we choose the range of the argument of $\alpha$ so that $0 \leq \arg (\alpha)<2 \pi$, and for any $\boldsymbol{y}=\left(y_{1}, y_{2}\right)^{t} \in \mathbb{R}^{2}$ we let $\arg (\boldsymbol{y}):=\arg \left(y_{1}+i y_{2}\right)$. Then we define

$$
\begin{array}{r}
S_{r}^{l}(\mathfrak{B}, X, y, \boldsymbol{\gamma}, \theta):=\operatorname{Card}\left\{\boldsymbol{\alpha}=\left(\alpha_{0}, \cdots, \alpha_{l-2}\right)^{t} \in\left(\mathfrak{B}^{-1}\right)^{l-1}:\left\|\boldsymbol{\alpha}^{(r)}\right\| \leq y,\right. \\
\left.H_{\infty}(\boldsymbol{\alpha}, \boldsymbol{\gamma}) \leq X, 0 \leq \arg \left(\alpha_{n-2}^{(r)}\right) \leq \theta\right\}
\end{array}
$$

for any $1 \leq r \leq r_{1}+r_{2}, y>0,0 \leq \theta \leq 2 \pi$ and $\gamma$ in $k^{s}, s \geq 1$. Suppose the values of $F(\boldsymbol{\alpha})$ depend only on $\left\|\boldsymbol{\alpha}^{(r)}\right\|$. Then

$$
\begin{aligned}
S^{l}(\mathfrak{B}, X, F) & =\sum_{\substack{\boldsymbol{\alpha} \in\left(\mathfrak{B}^{-1}\right)^{l-1} \\
H_{\infty}(\boldsymbol{\alpha}, 1) \leq X}} G\left(\left\|\boldsymbol{\alpha}^{(r)}\right\|\right) \\
& =\int_{0}^{\infty} G(y) d S_{r}^{l}(\mathfrak{B}, X, y, 1,2 \pi),
\end{aligned}
$$

where $G\left(\left\|\boldsymbol{\alpha}^{(r)}\right\|\right):=F(\boldsymbol{\alpha})$ and $G(y)$ is continuous on $(0, \infty)$. In order to estimate $S_{r}^{l}(\mathfrak{B}, X, y, \gamma, \theta)$, we follow the argument in [13] and first show that this is equal to the number of lattice points inside some domain in $\mathbb{R}^{l d}$. Then we use Theorems 4 and 5 in [13] to estimate the number of such lattice points. Lemma 5.3 below gives us an asymptotic estimate for $S_{r}^{l}(\mathfrak{B}, X, y, \gamma, \theta)$. Before we state the lemma, we need to introduce some notation. For any $\sigma \in \Sigma_{m}:=\left\{\left(c_{1}, c_{2}, \cdots, c_{m}\right): c_{j} \in\{1,2\}\right\}$ and $x>0$, we let

$$
D_{\sigma}(x):=\left\{\mathbf{u} \in \mathbb{R}^{m}: \prod_{j=1}^{m}\left(u_{j}^{2}+1\right)^{\frac{c_{j}}{2}} \leq x^{-1} \text { and } 0 \leq u_{j}, \forall j\right\}
$$

and

$$
f_{\sigma}^{l}(x):=x \int \ldots \int_{D_{\sigma}(x)} \prod_{j=1}^{m} u_{j}^{l c_{j}-1} d u_{j}
$$

for any $l \geq 1$. Note that if $x \geq 1$, then $f_{\sigma}^{l}(x)=0$ for any $\sigma$. For $1 \leq r \leq r_{1}+r_{2}$, we let

$$
\sigma_{r}:=\left(e_{1}, \cdots, e_{r-1}, e_{r+1}, \cdots, e_{r_{1}+r_{2}}\right)
$$


be an element in $\Sigma_{r_{1}+r_{2}-1}$. If $d=1$, i.e., $r_{1}=1$ and $r_{2}=0$, then we understand that

$$
f_{\sigma_{1}}^{l}(x)=x \chi_{[0,1]}(x)
$$

Let

$$
\mathbb{E}^{l d}:=\left\{\left(\boldsymbol{\alpha}_{1}, \boldsymbol{\alpha}_{2}, \cdots, \boldsymbol{\alpha}_{d}\right)^{t} \in \mathbb{R}^{l r_{1}} \times \mathbb{C}^{2 l r_{2}}: \boldsymbol{\alpha}_{r_{1}+i}=\overline{\boldsymbol{\alpha}_{r_{1}+r_{2}+i}}, 1 \leq i \leq r_{2}\right\},
$$

where $\boldsymbol{\alpha}_{i} \in \mathbb{R}^{l}$ for $1 \leq i \leq r_{1}$ and $\boldsymbol{\alpha}_{i} \in \mathbb{C}^{l}$ for $r_{1}+1 \leq i \leq d$. We then define $\rho: k^{l} \longrightarrow \mathbb{E}^{l d}$ by

and $\psi: \mathbb{E}^{l d} \longrightarrow \mathbb{R}^{l d}$ by

$$
\rho(\boldsymbol{\alpha}):=\left(\boldsymbol{\alpha}^{(1)}, \cdots, \boldsymbol{\alpha}^{(d)}\right)^{t}
$$

$$
\psi\left(\boldsymbol{\alpha}_{1}, \cdots, \boldsymbol{\alpha}_{d}\right):=\left(\boldsymbol{\beta}_{1}, \cdots, \boldsymbol{\beta}_{r_{1}+r_{2}}\right)^{t}
$$

where $\boldsymbol{\beta}_{i}:=\boldsymbol{\alpha}_{i}$ for $1 \leq i \leq r_{1}$,

$$
\boldsymbol{\beta}_{i}:=\left(\boldsymbol{\beta}_{i 0}, \cdots, \boldsymbol{\beta}_{i(l-1)}\right)^{t}
$$

for $r_{1}+1 \leq i \leq r_{1}+r_{2}$ and $\boldsymbol{\beta}_{i j}:=\left(\Re\left(\alpha_{i j}\right), \Im\left(\alpha_{i j}\right)\right)$ for $0 \leq j \leq l-1$. Note that $\psi \circ \rho$ embeds $k^{l}$ into $\mathbb{R}^{l d}$.

Next we suppose that $\Lambda$ is a lattice in $\mathbb{R}^{l}$. Let $\lambda_{1} \leq \cdots \leq \lambda_{l}$ be the successive minima of $\Lambda$ with respect to the unit ball in $\mathbb{R}^{l}$. Let $\left\{\boldsymbol{y}_{1}, \cdots, \overline{\boldsymbol{y}}_{l}\right\}$ be a corresponding set of linearly independent vectors in $\Lambda$ such that

$$
\left\|\boldsymbol{y}_{j}\right\|=\lambda_{j}
$$

for $1 \leq j \leq l$. Define

$$
\Lambda^{-i}:=\Lambda \cap \bigoplus_{j=1}^{l-i} \mathbb{R} \boldsymbol{y}_{j}
$$

for $1 \leq i \leq l-1$. Let $\boldsymbol{b}_{1}, \cdots, \boldsymbol{b}_{l}$ be a basis for $\Lambda$. If $\tau$ is some $m$-element subset of $\{1, \cdots, l\}$, then we write $V_{\tau}$ for the space spanned by the vectors $\boldsymbol{b}_{j}$ for $j \in \tau$ and $V_{\tau^{*}}$ for the space spanned by the remaining vectors $\boldsymbol{b}_{j}$. We also denote the orthogonal complement of a subspace $V$ in $\mathbb{R}^{l}$ by $V^{\perp}$, and the orthogonal projection of a domain $D$ onto $V$ by $D(V)$.

Lemma 5.3. Suppose $\mathfrak{B}$ is an integral ideal in $\mathfrak{O}_{k}$ and $1 \leq r \leq r_{1}+r_{2}$. Let $\boldsymbol{\gamma}$ be an element in $k^{s}, s \geq 1$, such that $1 \leq\left\|\gamma^{(j)}\right\|$ for any $1 \leq j \leq r_{1}+r_{2}$. For any $X \geq 1, l \geq 1$ and $y>0$ we let

$$
J=J(X, y, \gamma, r, l):=\int_{0}^{\frac{y}{\left\|\gamma^{(r)}\right\|}} f_{\sigma_{r}}^{l}\left(\frac{\left(u^{2}+1\right)^{\frac{e_{r}}{2}} H_{\infty}(\gamma)}{X}\right) \frac{u^{l e_{r}-1}}{\left(u^{2}+1\right)^{\frac{e_{r}}{2}}} d u .
$$

Then for any $0 \leq \theta \leq 2 \pi$, we have

$$
\begin{aligned}
& S_{r}^{l+1}(\mathfrak{B}, X, y, \gamma, \theta) \\
& \quad=c_{3} N(\mathfrak{B})^{l} \Delta J H_{\infty}(\gamma)^{l-1} X+O\left(N(\mathfrak{B})^{l-\frac{1}{d}} X \sum_{m=1}^{r_{1}+r_{2}} \sum_{\sigma \in \Sigma_{m}} f_{\sigma}^{l}\left(X^{-1}\right)\right),
\end{aligned}
$$

where the implicit constant depends only on $k$ and $l$,

$$
c_{3}=c_{3}(k, l)=(l V(l))^{r_{1}}(2 l V(2 l))^{r_{2}} 2^{l r_{2}}\left\|\Delta_{k}\right\|^{-\frac{l}{2}},
$$

and $\Delta=\Delta(\theta, r)$ is 1 or $\frac{\theta}{2 \pi}$ according as $1 \leq r \leq r_{1}$ or $r_{1}+1 \leq r \leq r_{1}+r_{2}$. 
DISTRIBUTION OF POINTS INPROJECTIVE SPACE OF BOUNDED HEIGHT 1097

Proof. We first suppose that $r_{1}+1 \leq r \leq r_{1}+r_{2}$. If we let $\Lambda:=\psi \circ \rho\left(\left(\mathfrak{B}^{-1}\right)^{l}\right)$ and for any $0 \leq \phi \leq 2 \pi$

$$
\begin{array}{r}
D(\phi):=\left\{\left(\boldsymbol{\beta}_{1}, \cdots, \boldsymbol{\beta}_{r_{1}+r_{2}}\right) \in \mathbb{R}^{l d}: \prod_{i=1}^{r_{1}+r_{2}}\left(\left\|\boldsymbol{\beta}_{i}\right\|^{2}+\left\|\boldsymbol{\gamma}^{(i)}\right\|^{2}\right)^{\frac{e_{i}}{2}} \leq X,\right. \\
\left.\left\|\boldsymbol{\beta}_{r}\right\| \leq y, 0 \leq \arg \left(\boldsymbol{\beta}_{r(l-1)}\right) \leq \phi\right\}
\end{array}
$$

where $\boldsymbol{\beta}_{i}=\left(\boldsymbol{\beta}_{i 0}, \cdots, \boldsymbol{\beta}_{i(l-1)}\right)^{t}$ and $\boldsymbol{\beta}_{i j} \in \mathbb{R}^{2}$ for $r_{1}+1 \leq i \leq r_{1}+r_{2}$ and $j=$ $0, \cdots, l-1$, then

$$
S_{r}^{l+1}(\mathfrak{B}, X, y, \gamma, \theta)=\operatorname{Card}\{\Lambda \cap D(\theta)\}
$$

by (5.15). In view of Lemma 1 in $[12], \rho\left(\left(\mathfrak{B}^{-1}\right)^{l}\right)$ is a lattice in $\mathbb{E}^{l d}$ and

$$
\operatorname{det}\left(\rho\left(\left(\mathfrak{B}^{-1}\right)^{l}\right)\right)=N\left(\mathfrak{B}^{-1}\right)^{l} \operatorname{det}\left(\rho\left(\mathfrak{O}_{k}^{l}\right)\right)=N\left(\mathfrak{B}^{-1}\right)^{l}\left\|\Delta_{k}\right\|^{\frac{l}{2}} .
$$

Since $\psi$ is a bijective linear transformation with determinant $2^{-l r_{2}}$, we have

$$
\operatorname{det}(\Lambda)=\left(N\left(\mathfrak{B}^{-1}\right) 2^{-r_{2}}\left\|\Delta_{k}\right\|^{\frac{1}{2}}\right)^{l}
$$

If we set $u_{i}=\left\|\boldsymbol{\beta}_{i}\right\|$ for $1 \leq i \leq r_{1}+r_{2}$ and use polar coordinates for each $\boldsymbol{\beta}_{i}$, then the $l d$-dimensional volume of $D(\theta)$ is

$$
\frac{\theta}{2 \pi}(l V(l))^{r_{1}}(2 l V(2 l))^{r_{2}} \int_{\substack{\prod_{i=1}^{r_{1}+r_{2}} \\\left(u_{i}^{2}+\left\|\gamma^{(i)}\right\|^{2}\right)^{e_{i} / 2} \leq X \\ 0 \leq u_{i}, \forall i}} \chi_{[0, y]}\left(u_{r}\right) \prod_{i=1}^{r_{1}+r_{2}} u_{i}^{l e_{i}-1} d u_{i}
$$

$$
=\frac{\theta}{2 \pi}(l V(l))^{r_{1}}(2 l V(2 l))^{r_{2}}\left(H_{\infty}(\gamma)\right)^{l} \int \ldots \int_{A}^{r_{1}+r_{2}} \prod_{i=1}^{l e_{i}-1} d u_{i}
$$

where

$$
A:=\left\{\mathbf{u} \in \mathbb{R}^{r_{1}+r_{2}}: 0 \leq u_{i}, \forall i, 0 \leq u_{r} \leq y /\left\|\gamma^{(r)}\right\|, \prod_{i=1}^{r_{1}+r_{2}}\left(u_{i}^{2}+1\right)^{\frac{e_{i}}{2}} \leq X / H_{\infty}(\gamma)\right\}
$$

In view of (5.17), we have

$$
\begin{aligned}
& \int \ldots \int_{A}^{r_{1}+r_{2}} \prod_{i=1}^{l e_{i}-1} d u_{i} \\
= & \frac{X}{H_{\infty}(\gamma)} \int_{0}^{\frac{y}{\left\|\gamma^{(r)}\right\|}} f_{\sigma_{r}}^{l}\left(\frac{\left(u^{2}+1\right)^{\frac{e_{r}}{2}} H_{\infty}(\gamma)}{X}\right) \frac{u^{l e_{r}-1}}{\left(u^{2}+1\right)^{\frac{e_{r}}{2}}} d u \\
= & J H_{\infty}(\gamma)^{-1} X .
\end{aligned}
$$


Now we apply Lemma 10 in [12] and Theorem 5 in [13], and in view of (5.20) and (5.21) we get

$$
\begin{aligned}
S_{r}^{l+1}(\mathfrak{B}, X, y, \gamma, \theta) & \\
= & \frac{\operatorname{Vol}(D(\theta))}{\operatorname{det}(\Lambda)}+O\left(\sum_{m=0}^{l d-1} \sum_{\tau} \frac{\operatorname{Vol}\left\{(D(\theta))\left(V_{\tau^{*}}^{\perp}\right)\right\}}{\operatorname{det}\left(\Lambda^{-(l d-m)}\right)}\right) \\
& =\frac{\operatorname{Vol}(D(\theta))}{\operatorname{det}(\Lambda)}+O\left(\sum_{m=0}^{l d-1} \frac{V_{m}(D(2 \pi))}{\operatorname{det}\left(\Lambda^{-(l d-m)}\right)}\right) \\
& =c_{3} N(\mathfrak{B})^{l} \Delta J H_{\infty}(\gamma)^{l-1} X+O\left(\sum_{m=0}^{l-1} \sum_{j=0}^{d-1} V_{m d+j}(D(2 \pi)) N(\mathfrak{B})^{m+\frac{j}{d}}\right),
\end{aligned}
$$

because $D(\theta) \subseteq D(2 \pi)$ and $D(2 \pi)$ is a coordinate domain. Next we analyze the error term in (5.22). If we replace all $\left\|\gamma^{(j)}\right\|$ by 1 and remove the conditions $\left\|\boldsymbol{\beta}_{r}\right\| \leq y$ and $0 \leq \arg \left(\boldsymbol{\beta}_{r(l-1)}\right) \leq 2 \pi$ in $D(2 \pi)$, then each summand in $V_{j}(D(2 \pi))$ becomes larger. As in proving (5.20), every summand in $V_{j}(D(2 \pi))$ is $\ll X f_{\sigma}^{l}\left(X^{-1}\right)$ for some $\sigma \in \Sigma_{m}$ and $1 \leq m \leq r_{1}+r_{2}$. Hence the error term in (5.22) is

$$
\ll N(\mathfrak{B})^{l-\frac{1}{d}} X \sum_{m=1}^{r_{1}+r_{2}} \sum_{\sigma \in \Sigma_{m}} f_{\sigma}^{l}\left(X^{-1}\right) .
$$

Therefore, (5.19) follows from (5.22) and (5.23). This proves (5.19) for $r_{1}+1 \leq$ $r \leq r_{1}+r_{2}$.

If $1 \leq r \leq r_{1}$, then the condition $0 \leq \arg \left(\alpha_{l-2}^{(r)}\right) \leq \theta$ can be removed because $\alpha_{l-2}^{(r)}$ is real. So, $S_{r}^{l+1}(\mathfrak{B}, X, y, \gamma, \theta)=S_{r}^{l+1}(\mathfrak{B}, X, y, \gamma, 2 \pi)$, and this proves (5.19) for $1 \leq r \leq r_{1}$.

It follows from the Dedekind-Weber theorem that for $y>0$,

$$
\sum_{\substack{\mathfrak{B} \subseteq \mathfrak{O}_{k} \\ N(\mathfrak{B}) \leq y}} 1=h \varkappa y+O\left(y^{1-\frac{1}{d}}\right),
$$

where

$$
\varkappa:=\frac{2^{r_{1}+r_{2}} \pi^{r_{2}} R}{\omega\left\|\Delta_{k}\right\|^{\frac{1}{2}}} .
$$

Lemma 5.4. Let $G:[0, \infty) \longrightarrow \mathbb{C}$ be bounded and differentiable, and let $\left\|G^{\prime}(t)\right\|$ be integrable on $[0, \infty)$. Suppose $\|G(t)\| \leq M$ and $\int_{0}^{\infty}\left\|G^{\prime}(t)\right\| d t \leq M^{\prime}$. Then for any $X \geq 1, l \geq 1$ and $1 \leq r \leq r_{1}+r_{2}$, we have

$$
\begin{aligned}
\sum_{\substack{\mathfrak{B} \subseteq \mathfrak{O}_{k} \\
N(\mathfrak{B}) \leq X}} S^{l+1}\left(\mathfrak{B}, X / N(\mathfrak{B}), G\left(\left\|\boldsymbol{\alpha}^{(r)}\right\|\right)\right) \\
\quad=c_{4} X^{l+1} \int_{0}^{\infty} G(y) \frac{y^{l e_{r}-1}}{\left(y^{2}+1\right)^{(l+1) \frac{e_{r}}{2}}} d y+O\left(\left(M+M^{\prime}\right) X^{l+1-\frac{1}{d}}\right),
\end{aligned}
$$


where the implicit constant depends only on $k, l$ and $G$, and

$$
c_{4}=c_{4}(k, l, r):=\frac{c_{3} h \varkappa}{l+1} \prod_{\substack{i \neq r \\ i=1}}^{r_{1}+r_{2}} \frac{\Gamma\left(\frac{e_{i}}{2}\right) \Gamma\left(\frac{l e_{i}}{2}\right)}{2 \Gamma\left(\frac{(l+1) e_{i}}{2}\right)} .
$$

Proof. We first suppose $d \geq 2$. By (5.16),

$$
S^{l+1}\left(\mathfrak{B}, X / N(\mathfrak{B}), G\left(\left\|\boldsymbol{\alpha}^{(r)}\right\|\right)\right)=\int_{0}^{\infty} G(y) d S_{r}^{l+1}(\mathfrak{B}, X / N(\mathfrak{B}), y, 1,2 \pi) .
$$

Hence from Lemma 5.3, we have

$$
\begin{aligned}
S^{l+1}\left(\mathfrak{B}, X / N(\mathfrak{B}), G\left(\left\|\boldsymbol{\alpha}^{(r)}\right\|\right)\right) \\
=c_{3} N(\mathfrak{B})^{l-1} X \int_{0}^{\infty} G(y) f_{\sigma_{r}}^{l}\left(\frac{\left(y^{2}+1\right)^{e_{r} / 2} N(\mathfrak{B})}{X}\right) \frac{y^{l e_{r}-1}}{\left(y^{2}+1\right)^{e_{r} / 2}} d y \\
\quad+\int_{0}^{\infty} G(y) d O\left(N(\mathfrak{B})^{l-1-\frac{1}{d}} X \sum_{m=1}^{r_{1}+r_{2}} \sum_{\sigma \in \Sigma_{m}} f_{\sigma}^{l}\left(N(\mathfrak{B}) X^{-1}\right)\right) .
\end{aligned}
$$

Next we consider the following summation which appears on the right hand side of (5.26) when we sum over $\mathfrak{B} \subseteq \mathfrak{O}_{k}, N(\mathfrak{B}) \leq X$. For simplicity, we temporarily fix $y \geq 0$ and let

$$
f(x)=f_{\sigma_{r}}^{l}\left(\frac{\left(y^{2}+1\right)^{e_{r} / 2} x}{X}\right),
$$

for $0 \leq x \leq X$. Then, by (5.24), we have

$$
\begin{aligned}
& \sum_{\substack{\mathfrak{B} \subseteq \mathfrak{O}_{k} \\
N(\mathfrak{B}) \leq X}} N(\mathfrak{B})^{l-1} f(N(\mathfrak{B}))=\int_{0}^{X} x^{l-1} f(x) d \sum_{\substack{\mathfrak{B} \subseteq \mathfrak{O}_{k} \\
N(\overline{\mathfrak{B}}) \leq x}} 1 \\
& \quad=h \varkappa \int_{0}^{X} x^{l-1} f(x) d x+O\left(\int_{0}^{X} x^{1-\frac{1}{d}}\left|\frac{d}{d x} x^{l-1} f(x)\right| d x\right),
\end{aligned}
$$

because $f(X)=0$ for any $\sigma$ and $y \geq 0$. For any $\sigma \in \Sigma_{m}, 1 \leq m \leq r_{1}+r_{2}$ and $0 \leq s<1$, by (5.17), we have

$$
\begin{array}{rl}
\int_{0}^{X} & f(x) x^{l-1-s} d x \\
& =\frac{\left(y^{2}+1\right)^{e_{r} / 2}}{X} \int_{0}^{X} x^{l-s} \int_{D_{\sigma}\left(\left(y^{2}+1\right)^{e_{r} / 2} x X^{-1}\right)^{2}} \ldots \prod_{i=1}^{m} u_{i}^{l c_{i}-1} d u_{i} d x \\
& =\left(y^{2}+1\right)^{e_{r} / 2} X^{l-s} \int_{0}^{\infty} \cdots \int_{0}^{\infty} \int_{0}^{\left(y^{2}+1\right)^{-e_{r} / 2} \prod_{i=1}^{m}\left(u_{i}^{2}+1\right)^{-c_{i} / 2}} x^{l-s} d x \prod_{i=1}^{m} u_{i}^{l c_{i}-1} d u_{i} \\
& =\frac{1}{l+1-s}\left\{\frac{X}{\left(y^{2}+1\right)^{\frac{e_{r}}{2}}}\right\}^{l-s} \prod_{i=1}^{m} \frac{\Gamma\left(l c_{i} / 2\right) \Gamma\left(c_{i}(1-s) / 2\right)}{2 \Gamma\left(c_{i}(l+1-s) / 2\right)}
\end{array}
$$


because $\int_{0}^{\infty} \frac{u^{2 q-1}}{\left(1+u^{2}\right)^{p+q}} d u=\frac{\Gamma(p) \Gamma(q)}{2 \Gamma(p+q)}$ for any $p, q>0$. In view of Lemma 13 in [12], we have $f_{\sigma}^{l}{ }^{\prime}(x) \leq 0$, and hence the error term in (5.27) is

$$
\ll \int_{0}^{X} f(x) x^{l-1-\frac{1}{d}} d x \ll\left(\frac{X}{\left(y^{2}+1\right)^{e_{r} / 2}}\right)^{l-\frac{1}{d}},
$$

by (5.28). Combining (5.27), (5.28) and (5.29), we now have, for any $y \geq 0$,

$$
\begin{aligned}
& \sum_{\substack{\mathfrak{B} \subseteq \mathfrak{O}_{k} \\
N(\mathfrak{B}) \leq X}} N(\mathfrak{B})^{l-1} f(N(\mathfrak{B})) \\
= & \frac{c_{4}}{c_{3}}\left(\frac{X}{\left(y^{2}+1\right)^{e_{r} / 2}}\right)^{l}+O\left(\left(\frac{X}{\left(y^{2}+1\right)^{e_{r} / 2}}\right)^{l-\frac{1}{d}}\right) .
\end{aligned}
$$

Thus by (5.26),

$$
\begin{aligned}
& \sum_{\substack{\mathfrak{B} \subseteq \mathfrak{O}_{k} \\
N(\mathfrak{B}) \leq X}} S^{l+1}\left(\mathfrak{B}, X / N(\mathfrak{B}), G\left(\left\|\boldsymbol{\alpha}^{(r)}\right\|\right)\right)=c_{4} X^{l+1} \int_{0}^{\infty} G(y) \frac{y^{l e_{r}-1}}{\left(y^{2}+1\right)^{\frac{(l+1) e_{r}}{2}} d y} \\
& +O\left(M X^{l+1-\frac{1}{d}}+\left(M+M^{\prime}\right) \sum_{\substack{\mathfrak{B} \subseteq \mathfrak{O}_{k} \\
N(\mathfrak{B}) \leq X}} N(\mathfrak{B})^{l-1-\frac{1}{d}} X \sum_{m=1}^{r_{1}+r_{2}} \sum_{\sigma \in \Sigma_{m}} f_{\sigma}^{l}\left(N(\mathfrak{B}) X^{-1}\right)\right) .
\end{aligned}
$$

Using an argument as in our proof of (5.30), we can show that the second error term in the last equation is $\ll\left(M+M^{\prime}\right) X^{l+1-\frac{1}{d}}$. This proves (5.25) for $d \geq 2$. The case $d=1$ can be proved in a similar manner.

For any $N \geq 2, n \geq 1, x \geq 1$ and $\boldsymbol{\beta}$ in $k^{N-1}$, we let

$$
T_{n}^{N}(x, \boldsymbol{\beta}):=\sum_{\substack{\mathfrak{B} \subseteq \mathfrak{O}_{k} \\ N(\mathfrak{B}) \leq x}} S^{N}\left(\mathfrak{B}, x / N(\mathfrak{B}), Q_{2 n}^{N, v}\left(\Delta_{v}((\boldsymbol{\alpha}, 1),(\boldsymbol{\beta}, 1))\right)\right)
$$

Since $v \mid \infty$, so $v$ corresponds to some embedding of $k$ into $\mathbb{C}$ such that

$$
\|\alpha\|_{v}=\left\|\alpha^{(r)}\right\|
$$

for any $\alpha \in k$. From now on, we fix $r$ to correspond to this embedding of $k$ into $k_{v}$.

We will prove Theorem 5.1 by induction on $N \geq 2$. The following lemma shows that it is true for $N=2$ and this starts our initial inductive step.

Lemma 5.5. Suppose $v \mid \infty$. Let $\beta$ be an element in $k$ and $n$ a positive integer. Then for any $X \geq 1$, we have

$$
T_{n}^{2}(X, \beta) \ll n^{5 n} X^{2-\frac{1}{d}}
$$

where the implicit constant depends only on $k$.

Proof. We first consider the case that $k_{v} \cong \mathbb{R}$. In this case, by (4.6),

$$
Q_{2 n}^{2, v}\left(\Delta_{v}((\alpha, 1),(\beta, 1))\right)=\alpha_{2 n}^{2} C_{2 n}^{0}\left(\sqrt{1-\Delta_{v}^{2}((\alpha, 1),(\beta, 1))}\right),
$$

and $\alpha^{(r)}$ is real for any $\alpha$ in $k$. Hence if we let $\theta$ and $\phi$ be the angles in $[0, \pi]$ such that

$$
\cos \theta:=\frac{\alpha^{(r)}}{\sqrt{1+\left\|\alpha^{(r)}\right\|^{2}}} \quad \text { and } \quad \cos \phi:=\frac{\beta^{(r)}}{\sqrt{1+\left\|\beta^{(r)}\right\|^{2}}}
$$


then

$$
\sqrt{1-\Delta_{v}^{2}((\alpha, 1),(\beta, 1))}=\frac{\left\|\alpha^{(r)} \beta^{(r)}+1\right\|}{\sqrt{1+\left\|\alpha^{(r)}\right\|^{2}} \sqrt{1+\left\|\beta^{(r)}\right\|^{2}}}=\|\cos (\theta-\phi)\| .
$$

Since $C_{m}^{0}(\cos \psi)=\frac{2}{m} \cos m \psi$ and $C_{m}^{1}(\cos \psi)=\frac{\sin (m+1) \psi}{\sin \psi}$, so we have

$$
C_{2 n}^{0}\left(\sqrt{1-\Delta_{v}^{2}((\alpha, 1),(\beta, 1))}\right)=C_{2 n}^{0}(\cos \theta) \cos 2 n \phi+\frac{1}{n} C_{2 n-1}^{1}(\cos \theta) \sin \theta \sin 2 n \phi .
$$

Because $C_{2 n-1}^{1}(x)$ is odd, when we sum over $\alpha \in \mathfrak{B}^{-1}$, the second term in (5.33) vanishes. So, from (4.6), (5.14), (5.31) and (5.33), we have

$$
\begin{aligned}
T_{n}^{2}(X, \beta) & =\alpha_{2 n}^{2}(\cos 2 n \phi) \sum_{\substack{\mathfrak{B} \subseteq \mathfrak{O}_{k} \\
N(\mathfrak{B}) \leq X}} S^{2}\left(\mathfrak{B}, X / N(\mathfrak{B}), G_{1}\left(\left\|\alpha^{(r)}\right\|\right)\right) \\
& \ll n\left|\sum_{\substack{\mathfrak{B} \subseteq \mathfrak{O}_{k} \\
N(\mathfrak{B}) \leq X}} S^{2}\left(\mathfrak{B}, X / N(\mathfrak{B}), G_{1}\left(\left\|\alpha^{(r)}\right\|\right)\right)\right|,
\end{aligned}
$$

where $G_{1}(t)=C_{2 n}^{0}\left(\frac{t}{\sqrt{t^{2}+1}}\right)$. By putting $G(t)=G_{1}(t)$ and $l=1$ in Lemma 5.4 and using

$$
\int_{0}^{\infty} G_{1}(t) \frac{1}{t^{2}+1} d t=0
$$

we get (5.32) from (5.25) because $\left\|G_{1}(t)\right\| \leq 1 / n$ and $\int_{0}^{\infty}\left\|G_{1}^{\prime}(t)\right\| d t \ll n$. This proves (5.32) for $k_{v} \cong \mathbb{R}$. Similarly, if $k_{v} \cong \mathbb{C}$, then by (4.7) and the fact that $P_{n}^{(0,0)}(x)=C_{n}^{\frac{1}{2}}(x)$, we have

$$
\begin{aligned}
Q_{2 n}^{2, v}\left(\Delta_{v}((\alpha, 1),(\beta, 1))\right) & =\beta_{2 n}^{2} P_{n}^{(0,0)}\left(2 \Delta_{v}^{2}((\alpha, 1),(\beta, 1))-1\right) \\
& =\beta_{2 n}^{2} C_{n}^{\frac{1}{2}}\left(2 \Delta_{v}^{2}((\alpha, 1),(\beta, 1))-1\right)
\end{aligned}
$$

and we let

$$
\alpha^{(r)}=\rho_{1} e^{i \psi_{1}} \quad \text { and } \quad \beta^{(r)}=\rho_{2} e^{i \psi_{2}}
$$

for $\rho_{1}, \rho_{2}>0$ and $\psi_{1}, \psi_{2} \in[0,2 \pi)$. We also let $\theta_{i} \in[0, \pi]$ be such that $\cos \theta_{i}=\frac{\rho_{i}^{2}-1}{\rho_{i}^{2}+1}$ for $i=1,2$. Thus, by the addition theorem (4.3),

$$
\begin{aligned}
C_{n}^{\frac{1}{2}}(1- & \left.2 \Delta_{v}^{2}((\alpha, 1),(\beta, 1))\right) \\
= & C_{n}^{\frac{1}{2}}\left(\cos \theta_{1} \cos \theta_{2}+\sin \theta_{1} \sin \theta_{2} \cos \left(\psi_{1}-\psi_{2}\right)\right) \\
= & C_{n}^{\frac{1}{2}}\left(\cos \theta_{1}\right) C_{n}^{\frac{1}{2}}\left(\cos \theta_{2}\right)+2 \sum_{j=1}^{n} b_{j, n}^{\frac{1}{2}} \sin ^{j} \theta_{1} \sin ^{j} \theta_{2} \\
& \quad \times C_{n-j}^{\frac{1}{2}+j}\left(\cos \theta_{1}\right) C_{n-j}^{\frac{1}{2}+j}\left(\cos \theta_{2}\right) \cos j\left(\psi_{1}-\psi_{2}\right) .
\end{aligned}
$$

Since $C_{j}^{0}(x)$ is odd if $j$ is odd, the contribution corresponding to the odd $j$ on the left hand side of (5.34) will be zero when we sum over $\alpha \in \mathfrak{B}^{-1}$. Hence from (4.4), 
(4.5) and (4.7), we have

$$
T_{n}^{2}(X, \beta) \ll n^{\frac{1}{2}}\left|\sum_{\substack{\mathfrak{B} \subseteq \mathfrak{O}_{k} \\ N(\mathfrak{B}) \leq X}} S^{2}\left(\mathfrak{B}, X / N(\mathfrak{B}), G_{2}\left(\left\|\alpha^{(r)}\right\|\right)\right)\right|+n^{\frac{1}{2}}(2 e n)^{2 n}
$$

$(5.35) \times \sum_{j=1}^{[n / 2]}\left|\sum_{\substack{\mathfrak{B} \subseteq \mathfrak{O}_{k} \\ N(\mathfrak{B}) \leq X}} \sum_{\substack{\alpha \in \mathfrak{B}^{-1} \\(\alpha, 1) \leq X / N(\mathfrak{B})}} H_{j}\left(\left\|\alpha^{(r)}\right\|\right) \cos 2 j\left(\arg \left(\alpha^{(r)}\right)-\psi_{2}\right)\right|$,

where $G_{2}(t)=C_{n}^{\frac{1}{2}}\left(\frac{t^{2}-1}{t^{2}+1}\right)$ and $H_{j}(t)=\left\{\frac{2 t}{t^{2}+1}\right\}^{2 j} C_{n-2 j}^{\frac{1}{2}+2 j}\left(\frac{t^{2}-1}{t^{2}+1}\right)$. By putting $G(t)=$ $G_{2}(t)$ and $l=1$ in Lemma 5.4, the first term on the right hand side of (5.35) is $\ll n^{\frac{5}{2}} X^{2-\frac{1}{d}}$ because

$$
\int_{0}^{\infty} G_{2}(t) \frac{t}{\left(t^{2}+1\right)^{2}} d t=0 .
$$

Next we consider the second term in the right hand side of (5.35). By Lemma 5.3, we have

$$
\begin{aligned}
& \sum_{\substack{\mathfrak{B} \subseteq \mathfrak{O}_{k} \\
N(\mathfrak{B}) \leq X}} \sum_{\substack{\alpha \in \mathfrak{B}^{-1},\left\|\alpha^{(r)}\right\| \leq y \\
H_{\infty}(\alpha, 1) \leq X / N(\mathfrak{B})}} \cos 2 j\left(\arg \left(\alpha^{(r)}\right)-\psi_{2}\right) \\
= & \sum_{\substack{\mathfrak{B} \subseteq \mathfrak{O}_{k} \\
N(\mathfrak{B}) \leq X}} \int_{0}^{2 \pi} \cos 2 j\left(\psi-\psi_{2}\right) d S_{r}^{2}(\mathfrak{B}, X / N(\mathfrak{B}), y, 1, \psi) \\
\ll & \sum_{\substack{\mathfrak{B} \subseteq \mathfrak{O}_{k} \\
N(\mathfrak{B}) \leq X}} N(\mathfrak{B})^{-\frac{1}{d}} X \sum_{m=1}^{r_{1}+r_{2}} \sum_{\sigma \in \Sigma_{m}} f_{\sigma}^{1}\left(N(\mathfrak{B}) X^{-1}\right) \\
\ll & X^{2-\frac{1}{d}} .
\end{aligned}
$$

Therefore the double summation over $\mathfrak{B}$ and $\alpha$ in the second term in (5.35) is equal to

$$
\begin{aligned}
& \int_{0}^{\infty} H_{j}(y) d \sum_{\substack{\mathfrak{B} \subseteq \mathfrak{O}_{k} \\
N(\mathfrak{B}) \leq X}} \sum_{\substack{\alpha \in \mathfrak{B}^{-1},\left\|\alpha^{(r)}\right\| \leq y \\
H_{\infty}(\alpha, 1) \leq X / N(\mathfrak{B})}} \cos 2 j\left(\arg \left(\alpha^{(r)}\right)-\psi_{2}\right) \\
\ll & j(e n)^{4 j+2} X^{2-\frac{1}{d}}
\end{aligned}
$$

by (4.5). This shows that the second term in (5.35) is $\ll n^{5 n} X^{2-\frac{1}{d}}$, and so proves (5.32) for $k_{v} \cong \mathbb{C}$.

Lemma 5.6. Suppose $v \mid \infty$ and $N \geq 2$. Let $\boldsymbol{\beta}$ be an element in $k^{N-1}, n$ a positive integer. Then for any $X \geq 1$,

$$
T_{n}^{N}(X, \boldsymbol{\beta}) \ll n^{9(N-1) n} X^{N-\frac{1}{d}},
$$

where the implicit constant depends only on $k$ and $N$.

Proof. We prove the lemma by induction on $N$. The case $N=2$ is true because of Lemma 5.5. Now we suppose the lemma is true for $N-1$ for $N \geq 3$. 
Let $k_{v} \cong \mathbb{R}$. Then

$$
\begin{aligned}
1-\Delta_{v}^{2}((\boldsymbol{\alpha}, 1),(\boldsymbol{\beta}, 1)) & =\frac{\left\|\sum_{i=0}^{N-1} \alpha_{i}^{(r)} \beta_{i}^{(r)}+1\right\|^{2}}{\left\|\left(\boldsymbol{\alpha}^{(r)}, 1\right)\right\|^{2} \cdot\left\|\left(\boldsymbol{\beta}^{(r)}, 1\right)\right\|^{2}} \\
& =\|\cos \theta \cos \phi+\sin \theta \sin \phi \cos \psi\|^{2}
\end{aligned}
$$

where $\cos \theta=\frac{\alpha_{0}^{(r)}}{\left\|\left(\boldsymbol{\alpha}^{(r)}, 1\right)\right\|}, \cos \phi=\frac{\beta_{0}^{(r)}}{\left\|\left(\boldsymbol{\beta}^{(r)}, 1\right)\right\|}$ and $\cos \psi=\frac{\sum_{i=1}^{N-1} \alpha_{i}^{(r)} \beta_{i}^{(r)}+1}{\left\|\left(\boldsymbol{\alpha}^{\prime(r)}, 1\right)\right\| \cdot\left\|\left(\boldsymbol{\beta}^{\prime(r)}, 1\right)\right\|}$ for $0 \leq \theta, \phi, \psi \leq \pi$ and $\boldsymbol{\alpha}^{\prime}=\left(\alpha_{1}, \cdots, \alpha_{N-2}\right), \boldsymbol{\beta}^{\prime}=\left(\beta_{1}, \cdots, \beta_{N-2}\right)$. So by $(4.3)$ and (4.6), we have

$$
\begin{aligned}
& Q_{2 n}^{N, v}\left(\Delta_{v}((\boldsymbol{\alpha}, 1),(\boldsymbol{\beta}, 1))\right) \\
= & \alpha_{2 n}^{N} C_{2 n}^{\frac{N-2}{2}}\left(\sqrt{1-\Delta_{v}^{2}((\boldsymbol{\alpha}, 1),(\boldsymbol{\beta}, 1))}\right) \\
= & \alpha_{2 n}^{N} \sum_{j=0}^{2 n} b_{j, 2 n}^{\frac{N-2}{2}} \sin ^{j} \theta \sin ^{j} \phi C_{2 n-j}^{\frac{N-2}{2}+j}(\cos \theta) C_{2 n-j}^{\frac{N-2}{2}+j}(\cos \phi) \frac{N-3+2 j}{N-3} C_{j}^{\frac{N-3}{2}}(\cos \psi),
\end{aligned}
$$

where $b_{0,2 n}^{\frac{N-2}{2}}=1$. Since $C_{2 n-j}^{\frac{N-2}{2}+j}(x)$ is odd if $j$ is odd, we get, as before,

$$
\begin{aligned}
& T_{n}^{N}(X, \boldsymbol{\beta}) \ll(5 e n)^{N-3+4 n} \sum_{j=0}^{n} \mid \sum_{\substack{\mathfrak{B} \subseteq \mathfrak{O}_{k} \\
N(\mathfrak{B}) \leq X}} \sum_{\begin{array}{c}
\boldsymbol{\alpha}^{\prime} \in\left(\mathfrak{B}^{-1}\right)^{N-2} \\
H_{\infty}\left(\boldsymbol{\alpha}^{\prime}, 1\right) \leq X / N(\mathfrak{B})
\end{array}} \\
& \times Q_{2 j}^{N-1, v}\left(\Delta_{v}\left(\left(\boldsymbol{\alpha}^{\prime}, 1\right),\left(\boldsymbol{\beta}^{\prime}, 1\right)\right)\right) \sum_{\substack{\alpha_{0} \in \mathfrak{B}^{-1} \\
H_{\infty}\left(\alpha_{0}, \boldsymbol{\alpha}^{\prime}, 1\right) \leq X / N(\mathfrak{B})}} F_{j}\left(\frac{\left\|\alpha_{0}^{(r)}\right\|}{\gamma_{r}}\right) \mid,
\end{aligned}
$$

where $\gamma_{i}:=\left\|\left(\boldsymbol{\alpha}^{\prime(i)}, 1\right)\right\|$ for $1 \leq i \leq r_{1}+r_{2}$ and $F_{j}(t)=\left\{\frac{1}{t^{2}+1}\right\}^{j} C_{2 n-2 j}^{\frac{N-2}{2}+2 j}\left(\frac{t}{\sqrt{t^{2}+1}}\right)$. Now using Lemma 5.3 and (4.5), the summation over $\alpha_{0}$ in (5.37) is

$$
\begin{aligned}
= & \int_{0}^{\infty} F_{j}(y) d S_{r}^{2}\left(\mathfrak{B}, X / N(\mathfrak{B}), y \gamma_{r},\left(\boldsymbol{\alpha}^{\prime}, 1\right), 2 \pi\right) \\
= & c_{3}(k, 1) X \int_{0}^{\infty} \frac{F_{j}(y)}{\left(y^{2}+1\right)^{\frac{1}{2}}} f_{\sigma_{r}}^{1}\left(\left(y^{2}+1\right)^{\frac{1}{2}} H_{\infty}\left(\boldsymbol{\alpha}^{\prime}, 1\right) N(\mathfrak{B}) X^{-1}\right) d y \\
& \quad+O\left((j+1)(2 e n)^{N-1+4 j} N(\mathfrak{B})^{-\frac{1}{d}} X \sum_{m=1}^{r_{1}+r_{2}} \sum_{\sigma \in \Sigma_{m}} f_{\sigma}^{1}\left(N(\mathfrak{B}) X^{-1}\right)\right) .
\end{aligned}
$$

So, in view of (5.37) and (5.38), we have

$$
\begin{aligned}
& \text { 5.39) } T_{n}^{N}(X, \boldsymbol{\beta}) \ll(5 e n)^{N-3+4 n} X \sum_{j=0}^{n}\left|\int_{0}^{\infty} \frac{F_{j}(y)}{\left(y^{2}+1\right)^{\frac{1}{2}}} \sum(y) d y\right| \\
& +(5 e n)^{2 N-4+8 n} \sum_{\substack{\mathfrak{B} \subseteq \mathfrak{O}_{k} \\
N(\mathfrak{B}) \leq X}} \sum_{\substack{\left.\boldsymbol{\alpha}^{\prime} \in(\mathfrak{B})^{-1}\right)^{N-2} \\
\left(\boldsymbol{\alpha}^{\prime}, 1\right) \leq X / N(\mathfrak{B})}} N(\mathfrak{B})^{-\frac{1}{d}} X \sum_{m=1}^{r_{1}+r_{2}} \sum_{\sigma \in \Sigma_{m}} f_{\sigma}^{1}\left(\frac{N(\mathfrak{B})}{X}\right),
\end{aligned}
$$


where

$$
\begin{gathered}
\sum(y)=\sum_{\substack{\mathfrak{B} \subseteq \mathfrak{O}_{k} \\
N(\mathfrak{B}) \leq X}} \sum_{\substack{\boldsymbol{\alpha}^{\prime} \in\left(\mathfrak{B}^{-1}\right)^{N-2} \\
H_{\infty}\left(\boldsymbol{\alpha}^{\prime}, 1\right) \leq X / N(\mathfrak{B})}} Q_{2 j}^{N-1, v}\left(\Delta_{v}\left(\left(\boldsymbol{\alpha}^{\prime}, 1\right),\left(\boldsymbol{\beta}^{\prime}, 1\right)\right)\right) \\
\times f_{\sigma_{r}}^{1}\left(\left(y^{2}+1\right)^{\frac{1}{2}} H_{\infty}\left(\boldsymbol{\alpha}^{\prime}, 1\right) N(\mathfrak{B}) X^{-1}\right) .
\end{gathered}
$$

For $j \geq 1$

$$
\begin{aligned}
\sum(y) & =\int_{0}^{1} f_{\sigma_{r}}^{1}\left(\left(y^{2}+1\right)^{\frac{1}{2}} x\right) d T_{j}^{N-1}\left(x X, \boldsymbol{\beta}^{\prime}\right) \\
& \ll(j+1)^{9(N-2) j} X^{N-1-\frac{1}{d}} \int_{0}^{1} x^{N-1-\frac{1}{d}}\left|\frac{d}{d x} f_{\sigma_{r}}^{1}\left(\left(y^{2}+1\right)^{\frac{1}{2}} x\right)\right| d x \\
& \ll(j+1)^{9(N-2) j}\left(\frac{X}{\left(y^{2}+1\right)^{\frac{1}{2}}}\right)^{N-1-\frac{1}{d}},
\end{aligned}
$$

by the inductive hypothesis, $f_{\sigma_{r}}{ }^{\prime}(x) \leq 0$ and (5.28). So for $j \geq 1$, we have

$$
\begin{aligned}
\int_{0}^{\infty} \frac{F_{j}(y)}{\left(y^{2}+1\right)^{\frac{1}{2}}} \sum(y) d y & \ll(j+1)^{9(N-2) j} X^{N-1-\frac{1}{d}} \int_{0}^{\infty} \frac{\left\|F_{j}(y)\right\|}{\left(y^{2}+1\right)^{\frac{N-\frac{1}{d}}{2}}} d y \\
& \ll(j+1)^{9(N-2) j}(2 e n)^{N-3+4 j} X^{N-1-\frac{1}{d}}
\end{aligned}
$$

by (4.5). By using Lemma 5.4 and the fact that

$$
\int_{0}^{\infty} \frac{F_{0}(y)}{\left(y^{2}+1\right)^{\frac{N}{2}}} d y=0
$$

we can show that (5.40) is also true for $j=0$. Hence the first term in the right side of (5.39) is $\ll n^{9(N-1) n} X^{N-\frac{1}{d}}$. Using a similar argument as in our proof of (5.30), we can show that the second term in the right hand side of (5.39) is also $\ll n^{9(N-1) n} X^{N-\frac{1}{d}}$. This proves (5.36) for $k_{v} \cong \mathbb{R}$. The proof in the case $k_{v} \cong \mathbb{C}$ is similar to the above proof but instead of using (4.3), we employ (4.2).

Proof of Theorem 5.1. First of all, by Dirichlet's Theorem, if we let $\tau \in k_{v}$ be such that $|\tau|_{v} \geq H^{\frac{d_{v}}{d}}$, then there is a $\boldsymbol{\beta}_{1} \in \mathbb{P}^{N-1}(k)$ such that

$$
\Delta_{v}\left(\boldsymbol{\beta}, \boldsymbol{\beta}_{1}\right) \ll|\tau|_{v}^{-\frac{d}{d_{v}}} \leq H^{-1},
$$

because $H(\boldsymbol{\beta}) \geq 1$. So,

$$
\begin{aligned}
& \sum_{\substack{\boldsymbol{\alpha} \in \mathbb{P}^{N-1}(k) \\
H(\boldsymbol{\alpha}) \leq H}} Q_{2 n}^{N, v}\left(\Delta_{v}(\boldsymbol{\alpha}, \underline{)})\right. \\
&= \sum_{\substack{\boldsymbol{\alpha} \in \mathbb{P}^{N-1}(k) \\
H(\boldsymbol{\alpha}) \leq H}} Q_{2 n}^{N, v}\left(\Delta_{v}\left(\boldsymbol{\alpha}, \boldsymbol{\beta}_{1}\right)\right)+O\left(n^{9(N-1) n} \sum_{\substack{\boldsymbol{\alpha} \in \mathbb{P}^{N-1}(k) \\
H(\boldsymbol{\alpha}) \leq H}} \Delta_{v}\left(\boldsymbol{\beta}, \boldsymbol{\beta}_{1}\right)\right) \\
&= \sum_{\substack{\boldsymbol{\alpha} \in \mathbb{P}^{N-1}(k) \\
H(\boldsymbol{\alpha}) \leq H}} Q_{2 n}^{N, v}\left(\Delta_{v}\left(\boldsymbol{\alpha}, \boldsymbol{\beta}_{1}\right)\right)+O\left(n^{9(N-1) n} H^{N d-1}\right) \\
&(H, 5),
\end{aligned}
$$

by (5.2) and (4.5). 
Without loss of generality, we may assume that $\boldsymbol{\beta}_{1}=\left(\beta_{0}, \cdots, \beta_{N-2}, 1\right)$. Hence from Lemmas 5.2 and 5.6

$$
\begin{aligned}
& \sum_{\substack{\boldsymbol{\alpha} \in \mathbb{P}^{N-1}(k) \\
H(\boldsymbol{\alpha}) \leq H}} Q_{2 n}^{N, v}\left(\Delta_{v}\left(\boldsymbol{\alpha}, \boldsymbol{\beta}_{1}\right)\right) \\
& =\sum_{\substack{\mathfrak{C} \subseteq \mathfrak{O}_{k} \\
N(\mathfrak{C}) \leq H^{d}}} \mu(\mathfrak{C}) T_{n}^{N}\left(H^{d} / N(\mathfrak{C}), \boldsymbol{\beta}_{1}\right)+O\left(n^{9(N-1) n} H^{(N-1) d}\right) \\
& \ll n^{9(N-1) n}\left(H^{N d-1} \sum_{\substack{\mathfrak{C} \subseteq \mathfrak{D}_{k} \\
N(\mathfrak{C}) \leq H^{d}}} \frac{\|\mu(\mathfrak{C})\|}{N(\mathfrak{C})^{N-\frac{1}{d}}}+H^{(N-1) d}\right) \\
& \ll n^{9(N-1) n}\left(H^{N d-1}+H \log H\right) .
\end{aligned}
$$

Therefore, (5.1) follows from this and (5.41). This completes the proof of Theorem 5.1 .

It remains to consider the case $v \nmid \infty$. In this case the orthonormal polynomials are unfamiliar and it is not known if there is an addition theorem for these polynomials. Of course this was essential to our argument establishing the inductive step in the proof of Theorem 5.1. Therefore in our proof of Theorem 2.2 for $v \nmid \infty$ we estimate the number of the rational points with low height inside a projective ball directly.

Theorem 5.7. Suppose $v \nmid \infty$. Let $\boldsymbol{\beta}$ be an element in $\mathbb{P}^{N-1}\left(k_{v}\right)$, and $m$ a positive integer. Then for any $H \geq 1$, we have

$$
\begin{aligned}
& \sum_{\substack{\boldsymbol{\alpha} \in \mathbb{P}^{N-1}(k) \\
H(\boldsymbol{\alpha}) \leq H}} \chi_{\mathbb{D}\left(\boldsymbol{\beta},\left\|\pi_{v}\right\|_{v}^{m}\right)}(\boldsymbol{\alpha}) \\
& \quad=c_{2}\left\|\pi_{v}\right\|_{v}^{m(N-1) d_{v}}\left\{\frac{1-\left\|\pi_{v}\right\|_{v}^{d_{v}}}{1-\left\|\pi_{v}\right\|_{v}^{N d_{v}}}\right\} H^{N d}+O\left(H^{N d-1}+H \log H\right),
\end{aligned}
$$

where $c_{2}$ is defined in (5.3) and the implicit constant depends only on $k$ and $N$.

Consequently Theorem 2.2 for $v \nmid \infty$ follows immediately from (2.12), (3.2), (5.2) and Theorem 5.7. Before we prove Theorem 5.7, we establish some preliminary lemmas.

Lemma 5.8. Suppose $v \nmid \infty$. Then for any $x \geq 1$, we have

$$
\sum_{\substack{\left(\mathfrak{B}, \mathfrak{P}_{v}\right)=1 \\ N(\mathfrak{B}) \leq x}} 1=h \varkappa\left(1-q_{v}^{-1}\right) x+O\left(x^{1-\frac{1}{d}}\right),
$$

where the summation is over all integral ideals in $\mathfrak{O}_{k}$, relatively prime to $\mathfrak{P}_{v}$, and having norm less than or equal to $x$.

Proof. Let $F(j)$ and $F^{*}(j)$ denote the number of integral ideals in $\mathfrak{O}_{k}$ with norm equal to $j$ and the number of integral ideals relatively prime to $\mathfrak{P}_{v}$ with norm equal to $j$, respectively. For any $s>1$, the Dedekind zeta function of $k$ is given by

$$
\zeta_{k}(s)=\sum_{\mathfrak{B} \subseteq \mathfrak{O}_{k}} N(\mathfrak{B})^{-s} .
$$


Then we have

$$
\zeta_{k}(s)=\sum_{j=1}^{\infty} \frac{F(j)}{j^{s}}
$$

and

$$
\sum_{\substack{\mathfrak{B} \subseteq \mathfrak{O}_{k} \\\left(\mathfrak{B}, \mathfrak{\mathfrak { P }}_{v}\right)=1}} N(\mathfrak{B})^{-s}=\sum_{j=1}^{\infty} \frac{F^{*}(j)}{j^{s}} .
$$

By the Euler product formula,

$$
\begin{aligned}
\sum_{j=1}^{\infty} \frac{F^{*}(j)}{j^{s}} & =\prod_{\mathfrak{P} \neq \mathfrak{P}_{v}}\left(1-N(\mathfrak{P})^{-s}\right)^{-1} \\
& =\zeta_{k}(s)\left(1-q_{v}^{-s}\right) \\
& =\sum_{j=1}^{\infty} \frac{F(j)}{j^{s}}+\sum_{j=1}^{\infty} \frac{F(j)}{\left(j q_{v}\right)^{s}}
\end{aligned}
$$

where the product is over all prime ideals in $\mathfrak{O}_{k}$ distinct from $\mathfrak{P}_{v}$. Hence, we have

$$
F^{*}(j)= \begin{cases}F(j) & \text { if } q_{v} \nmid j \\ F(j)-F\left(\frac{j}{q_{v}}\right) & \text { if } q_{v} \mid j\end{cases}
$$

Therefore,

$$
\begin{aligned}
\sum_{\substack{\left(\mathfrak{B}, \mathfrak{P}_{v}\right)=1 \\
N(\mathfrak{B}) \leq x}} 1 & =\sum_{j \leq x} F^{*}(j)=\sum_{j \leq x} F(j)-\sum_{j \leq \frac{x}{q_{v}}} F(j) \\
& =h \varkappa\left(1-q_{v}^{-1}\right) x+O\left(x^{1-\frac{1}{d}}\right)
\end{aligned}
$$

by (5.24). This proves Lemma 5.8 .

Lemma 5.9. Suppose $v \nmid \infty$. Let $\boldsymbol{\beta}=\left(\beta_{0}, \cdots, \beta_{N-2}\right)^{t}$ be an element in $k^{N-1}$ such that $\|(\boldsymbol{\beta}, 1)\|_{v}=1$ and $m \geq 1$. Then we have

$$
\Delta_{v}((\boldsymbol{\alpha}, 1),(\boldsymbol{\beta}, 1)) \leq\left\|\pi_{v}\right\|_{v}^{m}
$$

if and only if

$$
\left\|\alpha_{j}-\beta_{j}\right\|_{v} \leq\left\|\pi_{v}\right\|_{v}^{m}
$$

for $0 \leq j \leq N-2$ and any $\boldsymbol{\alpha}=\left(\alpha_{0}, \cdots, \alpha_{N-2}\right)^{t}$ in $k^{N-1}$.

Proof. We first note that

$$
\begin{aligned}
\left\|\alpha_{i} \beta_{j}-\alpha_{j} \beta_{i}\right\|_{v} & =\left\|\alpha_{i} \beta_{j}-\beta_{i} \beta_{j}+\beta_{i} \beta_{j}-\alpha_{j} \beta_{i}\right\|_{v} \\
& \leq \max \left\{\left\|\beta_{j}\right\|_{v}\left\|\alpha_{i}-\beta_{i}\right\|_{v},\left\|\beta_{i}\right\|_{v}\left\|\alpha_{j}-\beta_{j}\right\|_{v}\right\} \\
& \leq \max \left\{\left\|\alpha_{i}-\beta_{i}\right\|_{v},\left\|\alpha_{j}-\beta_{j}\right\|_{v}\right\} .
\end{aligned}
$$

Also, if $\left\|\alpha_{i}-\beta_{i}\right\|_{v} \leq\left\|\pi_{v}\right\|_{v}^{m}$, then $\|(\boldsymbol{\alpha}, 1)\|=1$. This proves the "if" part.

Suppose $\Delta_{v}((\boldsymbol{\alpha}, 1),(\boldsymbol{\beta}, 1)) \leq\left\|\pi_{v}\right\|_{v}^{m}$; then we claim that $\|(\boldsymbol{\alpha}, 1)\|_{v}=1$. For, if $\|(\boldsymbol{\alpha}, 1)\|_{v}>1$, then there is $j_{0}$ such that $\|\boldsymbol{\alpha}\|_{v}=\left\|\alpha_{j_{0}}\right\|_{v}>1$. Hence

$$
\begin{aligned}
\Delta_{v}((\boldsymbol{\alpha}, 1),(\boldsymbol{\beta}, 1)) & \geq \frac{\left\|\alpha_{j_{0}}-\beta_{j_{0}}\right\|_{v}}{\left\|\alpha_{j_{0}}\right\|_{v}} \\
& =1 .
\end{aligned}
$$


This contradicts $\Delta_{v}((\boldsymbol{\alpha}, 1),(\boldsymbol{\beta}, 1)) \leq\left\|\pi_{v}\right\|_{v}^{m}<1$. Therefore $\|(\boldsymbol{\alpha}, 1)\|_{v}=1$, and hence

$$
\left\|\alpha_{j}-\beta_{j}\right\|_{v} \leq \Delta_{v}((\boldsymbol{\alpha}, 1),(\boldsymbol{\beta}, 1)) \leq\left\|\pi_{v}\right\|_{v}^{m}
$$

for any $0 \leq j \leq N-2$. This completes the proof of the lemma.

Lemma 5.10. Let $m$ be a positive integer and $\beta$ an element in $\mathfrak{O}_{k}$. Suppose $v$ is a finite place of $k$ and $\mathfrak{B}$ is an integral ideal in $\mathfrak{O}_{k}$ such that

$$
\mathfrak{B}=\prod_{w \nmid \infty} \mathfrak{P}_{w}^{m_{w}},
$$

where $m_{w} \geq 0$ and $m_{w}=0$ for all but finitely many $w$. Then we have

$$
\left\{\alpha \in \mathfrak{B}^{-1}:\|\alpha-\beta\|_{v} \leq\left\|\pi_{v}\right\|_{v}^{m}\right\}=\mathfrak{P}_{v}^{m} \prod_{w \neq v} \mathfrak{P}_{w}^{-m_{w}}+\beta .
$$

Proof. Suppose $\alpha \in \mathfrak{P}_{v}^{m} \prod_{w \neq v} \mathfrak{P}_{w}^{-m_{w}}+\beta \subseteq \mathfrak{B}^{-1}$. Then we let $\alpha=\gamma+\beta$ for some $\gamma \in \mathfrak{P}_{v}^{m} \prod_{w \neq v} \mathfrak{P}_{w}^{-m_{w}}$, so that $\|\gamma\|_{v} \leq\left\|\pi_{v}\right\|_{v}^{m}$. Thus,

$$
\|\alpha-\beta\|_{v}=\|\gamma\|_{v} \leq\left\|\pi_{v}\right\|_{v}^{m}
$$

Conversely, suppose $\alpha \in \mathfrak{B}^{-1}$ and $\|\alpha-\beta\|_{v} \leq\left\|\pi_{v}\right\|_{v}^{m}$. Since $\beta \in \mathfrak{O}_{k} \subseteq \mathfrak{B}^{-1}$, it follows that $\alpha-\beta$ belongs to $\mathfrak{B}^{-1} \cap \mathfrak{P}_{v}^{m}$, which is equal to $\mathfrak{P}_{v}^{m} \prod_{w \neq v} \mathfrak{P}_{w}^{-m_{w}}$. This completes the proof of the lemma.

Proof of Theorem 5.7. The proof is similar to the proof of Theorem 5.1. First of all we show that we can assume $\boldsymbol{\beta} \in \mathbb{P}^{N-1}(k)$. In view of Dirichlet's Theorem, if $\boldsymbol{\beta}_{1} \in \mathbb{P}^{N-1}\left(k_{v}\right)$ and $\epsilon>0$, then there is $\boldsymbol{\beta}_{2}$ in $\mathbb{P}^{N-1}(k)$ such that

$$
\Delta_{v}\left(\boldsymbol{\beta}_{1}, \boldsymbol{\beta}_{2}\right)<\epsilon
$$

So, if $0<\epsilon<\left\|\pi_{v}\right\|_{v}^{m-1}\left(1-\left\|\pi_{v}\right\|_{v}\right)$, then

$$
\mathbb{D}\left(\boldsymbol{\beta}_{1},\left\|\pi_{v}\right\|_{v}^{m}\right)=\mathbb{D}\left(\boldsymbol{\beta}_{2},\left\|\pi_{v}\right\|_{v}^{m}\right) .
$$

Hence, without loss of generality, we may assume that $\boldsymbol{\beta}=\left(\boldsymbol{\beta}^{\prime}, 1\right)$ where $\boldsymbol{\beta}^{\prime}$ is in $k^{N-1}$ and $\|\boldsymbol{\beta}\|_{v}=1$. If we let

$$
\mathfrak{B}=\prod_{w \nmid \infty} \mathfrak{P}_{w}^{m_{w}} \quad\left(m_{w} \geq 0\right)
$$

be an integral ideal in $\mathfrak{O}_{k}$, then in view of Lemmas 5.9 and 5.10 , for $x \geq 1$,

$$
\begin{aligned}
& S^{N}\left(\mathfrak{B}, x, \chi_{\mathbb{D}}\left(\left(\boldsymbol{\beta}^{\prime}, 1\right),\left\|\pi_{v}\right\|_{v}^{m}\right)\right)=\sum_{\boldsymbol{\alpha} \in\left(\mathfrak{B}^{-1}\right)^{N-1}, H_{\infty}(\boldsymbol{\alpha}, 1) \leq x} 1 \\
& \Delta_{v}\left((\boldsymbol{\alpha}, 1),\left(\boldsymbol{\beta}^{\prime}, 1\right)\right) \leq\left\|\pi_{v}\right\|_{v}^{m} \\
& =\sum_{\boldsymbol{\alpha} \in\left(\mathfrak{B}^{-1}\right)^{N-1}, H_{\infty}(\boldsymbol{\alpha}, 1) \leq x} 1 \\
& \left\|\boldsymbol{\alpha}-\boldsymbol{\beta}^{\prime}\right\|_{v} \leq\left\|\pi_{v}\right\|_{v}^{m} \\
& =\sum_{\substack{\boldsymbol{\alpha} \in\left(\mathfrak{B}^{-1} \cap \mathfrak{P}_{v}^{m}\right)^{N-1}+\boldsymbol{\beta}^{\prime}, H_{\infty}(\boldsymbol{\alpha}, 1) \leq x}} 1 .
\end{aligned}
$$


We now apply the embeddings $\rho$ and $\psi$ defined in this section. Using the remark after Theorem 5 in [13], we get

$$
\begin{array}{r}
S^{N}\left(\mathfrak{B}, x, \chi_{\mathbb{D}\left(\boldsymbol{\beta},\left\|\pi_{v}\right\|_{v}^{m}\right)}\right)=\sum_{\boldsymbol{y} \in\left(\Lambda+\psi \circ \rho\left(\boldsymbol{\beta}^{\prime}\right)\right) \cap D_{x}} 1 \\
=\frac{\operatorname{Vol}\left(D_{x}\right)}{\operatorname{det}(\Lambda)}+O\left(\sum_{l=0}^{(N-1) d-1} \frac{V_{l}\left(D_{x}\right)}{\operatorname{det}\left(\Lambda^{-((N-1) d-l)}\right)}\right),
\end{array}
$$

where $\Lambda=\psi \circ \rho\left(\left(\mathfrak{B}^{-1} \cap \mathfrak{P}_{v}^{m}\right)^{N-1}\right)$ is a lattice in $\mathbb{R}^{(N-1) d}$ and

$$
D_{x}:=\left\{\boldsymbol{y} \in \mathbb{R}^{(N-1) d}: \prod_{j=1}^{r_{1}+r_{2}}\left(\left\|\boldsymbol{y}_{j}\right\|^{2}+1\right)^{\frac{e_{j}}{2}} \leq x\right\} .
$$

Using Lemma 1 in [12] and the multiplicity of the norm, we have

$$
\begin{aligned}
\operatorname{det}(\Lambda) & =\left\{N\left(\mathfrak{B}^{-1} \cap \mathfrak{P}_{v}^{m}\right) 2^{-r_{2}}\left\|\Delta_{k}\right\|^{\frac{1}{2}}\right\}^{N-1} \\
& =\left\{q_{v}^{m+m_{v}} 2^{-r_{2}} N(\mathfrak{B})^{-1}\left\|\Delta_{k}\right\|^{\frac{1}{2}}\right\}^{N-1} .
\end{aligned}
$$

As before,

$\operatorname{Vol}\left(D_{x}\right)$

$$
\begin{aligned}
& =((N-1) V(N-1))^{r_{1}}((2 N-2) V(2 N-2))^{r_{2}} \int_{D_{\sigma}\left(x^{-1}\right)} \ldots \int_{j=1}^{r_{1}+r_{2}} \prod_{j}^{(N-1) e_{j}-1} d u_{j} \\
& =((N-1) V(N-1))^{r_{1}}((2 N-2) V(2 N-2))^{r_{2}} x f_{\sigma}^{N-1}\left(x^{-1}\right),
\end{aligned}
$$

where $\sigma=\left(e_{1}, \cdots, e_{r_{1}+r_{2}}\right)$ and $f_{\sigma}^{N-1}(x)$ is defined in (5.17).

Combining (5.43)-(5.45), we have

$$
\begin{aligned}
& \sum_{\substack{\mathfrak{B} \subseteq \mathfrak{O}_{k} \\
N(\overline{\mathfrak{B}}) \leq x}} S^{N}\left(\mathfrak{B}, x / N(\mathfrak{B}), \chi_{\mathbb{D}}\left(\boldsymbol{\beta},\left\|\pi_{v}\right\|_{v}^{m}\right)\right) \\
= & c_{5} \frac{x}{q_{v}^{(N-1) m}} \sum_{\substack{\mathfrak{B} \subseteq \mathfrak{O}_{k} \\
N(\overline{\mathfrak{B}}) \leq x}} \frac{N(\mathfrak{B})^{N-2} f_{\sigma}^{N-1}(N(\mathfrak{B}) / x)}{q_{v}^{(N-1) m_{v}}}+O\left(x^{N-\frac{1}{d}}\right) \\
= & c_{5} \frac{x}{q_{v}^{(N-1) m}} \sum_{0 \leq l \leq \log x} \frac{1}{q_{v}^{l}} \\
\times & \sum_{\substack{\left(\mathfrak{B}, \mathfrak{P}_{v}\right)=1 \\
N(\mathfrak{B}) \leq x / q_{v}^{l}}} N(\mathfrak{B})^{N-2} f_{\sigma}^{N-1}\left(\frac{N(\mathfrak{B}) q_{v}^{l}}{x}\right)+O\left(x^{N-\frac{1}{d}}\right),
\end{aligned}
$$

where

$$
c_{5}=c_{5}(N, k):=2^{(N-1) r_{2}}\left\|\Delta_{k}\right\|^{-\frac{N-1}{2}}((N-1) V(N-1))^{r_{1}}((2 N-2) V(2 N-2))^{r_{2}}
$$

and the error term is treated as in (5.30). 
We now need to estimate the summation over $\mathfrak{B}$ on the right hand side of (5.47). By Lemma 5.8 , this summation over $\mathfrak{B}$ is

$$
\begin{aligned}
&= \int_{0}^{\frac{x}{q_{v}^{l}}} t^{N-2} f_{\sigma}^{N-1}\left(\frac{t q_{v}^{l}}{x}\right) d \sum_{\substack{\left(\mathfrak{B}, \mathfrak{P}_{v}\right)=1 \\
N(\mathfrak{B}) \leq t}} 1 \\
&=h \varkappa\left(1-q_{v}^{-1}\right) \int_{0}^{\frac{x}{q_{v}^{l}}} t^{N-2} f_{\sigma}^{N-1}\left(\frac{t q_{v}^{l}}{x}\right) d t \\
& \quad+O\left(\int_{0}^{\frac{x}{q_{v}^{l}}} t^{1-\frac{1}{d}}\left|\frac{d}{d t} t^{N-2} f_{\sigma}^{N-1}\left(\frac{t q_{v}^{l}}{x}\right)\right| d t\right) .
\end{aligned}
$$

Using (5.28), the error term in $(5.48)$ is $\ll\left(\frac{x}{q_{v}^{l}}\right)^{N-1-\frac{1}{d}}$ and the first integral in (5.48) is equal to

$$
\left(\frac{x}{q_{v}^{l}}\right)^{N-1} \cdot \frac{1}{N} \cdot\left(\frac{N V(N)}{2(N-1) V(N-1)}\right)^{r_{1}} \cdot\left(\frac{N V(2 N)}{2 \pi(N-1) V(2 N-2)}\right)^{r_{2}} .
$$

Hence by (5.3), (5.47) and (5.48)

$$
\begin{aligned}
& \sum_{\substack{\mathfrak{B} \subseteq \mathfrak{O}_{k} \\
N(\mathfrak{B}) \leq x}} S^{N}\left(\mathfrak{B}, x / N(\mathfrak{B}), \chi_{\mathbb{D}}\left(\boldsymbol{\beta},\left\|\pi_{v}\right\|_{v}^{m}\right)\right. \\
= & c_{2} \zeta_{k}(N)\left(1-q_{v}^{-1}\right) \frac{x^{N}}{q_{v}^{(N-1) m}} \sum_{0 \leq l \leq \frac{\log x}{\log q_{v}}} q_{v}^{-N l}+O\left(x^{N-\frac{1}{d}}\right) \\
= & c_{2} \zeta_{k}(N)\left(1-q_{v}^{-1}\right)\left(1-q_{v}^{-N}\right)^{-1} \frac{x^{N}}{q_{v}^{(N-1) m}}+O\left(x^{N-\frac{1}{d}}\right) .
\end{aligned}
$$

Therefore, by Lemma 5.2,

$$
\begin{gathered}
\sum_{\substack{\boldsymbol{\alpha} \in \mathbb{P}^{N-1}(k) \\
H(\boldsymbol{\alpha}) \leq H}} \chi_{\mathbb{D}\left(\boldsymbol{\beta},\left\|\pi_{v}\right\|_{v}^{m}\right)}(\boldsymbol{\alpha}) \\
=H^{N d} c_{2} \zeta_{k}(N)\left\|\pi_{v}\right\|_{v}^{m(N-1) d_{v}}\left\{\frac{1-\left\|\pi_{v}\right\|_{v}^{d_{v}}}{1-\left\|\pi_{v}\right\|_{v}^{N d_{v}}}\right\} \sum_{\substack{\mathfrak{C} \subseteq \mathfrak{D}_{k} \\
N(\mathfrak{C}) \leq H^{d} q}} \frac{\mu(\mathfrak{C})}{N(\mathfrak{C})^{N}} \\
\quad+O\left(H^{N d-1}+H \log H\right) \\
=c_{2}\left\|\pi_{v}\right\|_{v}^{m(N-1) d_{v}}\left\{\frac{1-\left\|\pi_{v}\right\|_{v}^{d_{v}}}{1-\left\|\pi_{v}\right\|_{v}^{N d_{v}}}\right\} H^{N d}+O\left(H^{N d-1}+H \log H\right),
\end{gathered}
$$

because $q_{v}=\left\|\pi_{v}\right\|_{v}^{-d_{v}}$. This proves (5.42), and completes the proof of Theorem 5.7 .

Next, we estimate the discrepancy. For any sequence $\left\{\boldsymbol{\alpha}_{l}\right\}_{l=1}^{\infty}$ in $\mathbb{P}^{N-1}\left(k_{v}\right)$ and $L \geq 1$, we define the discrepancy for $\left\{\boldsymbol{\alpha}_{l}\right\}_{l=1}^{\infty}$ to be

$$
D_{L}:=\sup \left\{\left|\frac{1}{L} \sum_{l=1}^{L} \boldsymbol{\chi}_{(\boldsymbol{\beta}, r)}\left(\boldsymbol{\alpha}_{l}\right)-\int_{\mathbb{P}^{N-1}\left(k_{v}\right)} \boldsymbol{\chi}_{(\boldsymbol{\beta}, r)}(\boldsymbol{\alpha}) d \mu_{v}(\boldsymbol{\alpha})\right|\right\} .
$$

where the supremum is taken over all $\boldsymbol{\beta} \in \mathbb{P}^{N-1}\left(k_{v}\right)$ and $0 \leq r \leq 1$. 
If $k_{v} \cong \mathbb{R}$, then using Lemma 3.4 with $\epsilon=M^{-\frac{2}{3}}$, Theorem 3.3, (4.5) and Lemmas 3.3.3, 4.2.2 in [4], we can show that

$$
D_{L} \ll M^{-\frac{1}{3}}+M^{\frac{N-2}{2}} \sum_{m=1}^{[M / 2]}\left|\frac{1}{L} \sum_{l=1}^{L} Q_{2 n}^{N, v}\left(\Delta_{v}\left(\boldsymbol{\alpha}_{l}, \boldsymbol{\beta}\right)\right)\right| .
$$

Similarly, if $k_{v} \cong \mathbb{C}$, then we have

$$
D_{L} \ll M^{-\frac{1}{2}}+\sum_{m=1}^{[M / 2]}\left|\frac{1}{L} \sum_{l=1}^{L} Q_{2 n}^{N, v}\left(\Delta_{v}\left(\boldsymbol{\alpha}_{l}, \boldsymbol{\beta}\right)\right)\right| .
$$

In particular, we consider the set of all rational points with low height, and for any $H \geq 1$ we let

$$
D_{H}^{*}:=\sup \left\{\left|\sum_{\substack{\boldsymbol{\alpha} \in \mathbb{P}^{N-1}(k) \\ H(\boldsymbol{\alpha}) \leq H}} \chi_{(\boldsymbol{\beta}, r)}(\boldsymbol{\alpha}) / \sum_{\substack{\boldsymbol{\alpha} \in \mathbb{P}^{N-1}(k) \\ H(\boldsymbol{\alpha}) \leq H}} 1-\int_{\mathbb{P}^{N-1}\left(k_{v}\right)} \boldsymbol{\chi}_{(\boldsymbol{\beta}, r)}(\boldsymbol{\alpha}) d \mu_{v}(\boldsymbol{\alpha})\right|\right\}
$$

where the supremum is taken over all $\boldsymbol{\beta} \in \mathbb{P}^{N-1}\left(k_{v}\right)$ and $0 \leq r \leq 1$.

Theorem 5.11. Let $H \geq 1$. For $v \mid \infty$, we have

$$
D_{H}^{*} \ll\left\{\frac{\log \log H}{\log H}\right\}^{\frac{1}{3}}
$$

for $k_{v} \cong \mathbb{R}$ and

$$
D_{H}^{*} \ll\left\{\frac{\log \log H}{\log H}\right\}^{\frac{1}{2}}
$$

for $k_{v} \cong \mathbb{C}$. For $v \nmid \infty$, we have

$$
D_{H}^{*} \ll H^{-1}+\frac{\log H}{H^{N d-1}}
$$

Proof. If we let $M=\frac{\log H}{10(N-1) \log \log H},(5.51)$ and (5.52) follow from (5.49),(5.50) and Theorem 5.1. Finally, in view of (3.5), (5.53) follows from Theorem 5.7.

We make one final remark here concerning our choice of height function. If we use the Weil height, in which the $l^{\infty}$-norm is used at the infinite places in (1.1) rather than the $l^{2}$-norm, then the set of rational points with low Weil height is no longer $\mu_{v}^{N}$-uniformly distributed. This suggests that the height defined in (1.8) is more natural in the projective setting.

The author wishes to thank Professor J. Vaaler for his valuable advice about this paper.

\section{REFERENCES}

[1] E. Bombieri, A.J. van der Poorten and J. D. Vaaler. Effective Measures of Irrationality for Cubic Extensions of Number Fields, Ann Scuola Norm. Sup. Pisa Cl. Sci. (4) 23 (1996), 211-248. MR 98d:11083

[2] E. Bombieri and J. Vaaler. On Siegel's Lemma, Invent. Math. 73, 11-32 (1983). MR 85g:11049a

[3] J.W.S. Cassels. Local Fields. (LMSST 3) Cambridge Univ. Press (1986). MR 87i:11172

[4] K.K. Choi. Diophantine Approximation on Projective Spaces over Number Fields, Ph. D. Dissertation. The University of Texas at Austin (1996). 
[5] K.K. Choi and J. D. Vaaler. Diophantine Approximation in Projective Space, Submitted for publication.

[6] S. Lang. Fundamentals of Diophantine Geometry, Springer-Verlag, New York, 1983. MR 85j: 11005

[7] P. Grabner. Erdös-Turán Type Discrepancy Bounds, Monatsh. Math. 111, 127-135 (1991). MR 92f: 11108

[8] L. Kuipers and H. Niederreiter. Uniform Distribution of Sequences. John Wiley \& Sons (1974). MR 54:7415

[9] R.S. Rumely. Capacity Theory on Algebraic Curves. Lecture Notes in Mathematics, vol. 1378, Springer-Verlag, New York, 1989. MR 91b:14018

[10] S. Schanuel. Heights in Number Fields, Bull. Soc. Math. France 107 (1979), 433-449. MR 81c: 12025

[11] G. Szegö. Orthogonal Polynomials. Colloquium Publications Vol. 23, Amer. Math. Soc. (1991). MR 51:8724

[12] J. Thunder. An Asymptotic Estimate for Heights of Algebraic Subspaces, Trans. Amer. Math. Soc. 331, 395-424 (1992). MR 92g: 11062

[13] J. Thunder. The Number of Solutions of Bounded Height to a System of Linear Equations, J. Number Theory 43, 228-250 (1993). MR 94a:11045

[14] S. Tyler. The Lagrange Spectrum in Projective Space over a Local Field, Ph. D. Dissertation. The University of Texas at Austin (1994).

[15] J. D. Vaaler. Some Extremal Functions in Fourier Analysis, Bull. Amer. Math. Soc. 12, 183-216 (1985). MR 86g:42005

Department of Mathematics, Statistics, Simon Fraser University, Burnaby, British Columbia, V5A 1S6, Canada

Department of Mathematics, University of British Columbia, Vancouver, British Columbia, V6T 1Z2, Canada

Current address: Department of Mathematics, The University of Hong Kong, Pokfulam Road, Hong Kong

E-mail address: choi@maths.hku.hk 